\title{
Endophilin A1 regulates dendritic spine morphogenesis and stability through interaction with p140Cap
}

\author{
Yanrui Yang ${ }^{1,2}$, Mengping $\mathrm{Wei}^{3,4}$, Ying Xiong ${ }^{5}$, Xiangyang $\mathrm{Du}^{1,2,6}$, Shaoxia Zhu ${ }^{1,2}$, Lin Yang ${ }^{1}$, Chen Zhang ${ }^{3,4}$, \\ Jia-Jia Liu ${ }^{1,2}$ \\ ${ }^{1}$ State Key Laboratory of Molecular Developmental Biology, Institute of Genetics and Developmental Biology, ${ }^{2}$ CAS Center for \\ Excellence in Brain Science, Chinese Academy of Sciences, Beijing 100101, China; ${ }^{3}$ State Key Laboratory of Biomembrane and \\ Membrane Biotechnology, School of Life Sciences, ${ }^{4}$ PKU-IDG/McGovern Institute for Brain Research, Peking University, Beijing \\ 100871, China; ${ }^{5}$ School of Life Sciences, University of Science and Technology of China, Hefei, Anhui 230026, China; ${ }^{6}$ University \\ of Chinese Academy of Sciences, Beijing 100039, China
}

Dendritic spines are actin-rich membrane protrusions that are the major sites of excitatory synaptic input in the mammalian brain, and their morphological plasticity provides structural basis for learning and memory. Here we report that endophilin A1, with a well-established role in clathrin-mediated synaptic vesicle endocytosis at the presynaptic terminal, also localizes to dendritic spines and is required for spine morphogenesis, synapse formation and synaptic function. We identify p140Cap, a regulator of cytoskeleton reorganization, as a downstream effector of endophilin A1 and demonstrate that disruption of their interaction impairs spine formation and maturation. Moreover, we demonstrate that knockdown of endophilin A1 or p140Cap impairs spine stabilization and synaptic function. We further show that endophilin A1 regulates the distribution of p140Cap and its downstream effector, the F-actin-binding protein cortactin as well as $\mathrm{F}$-actin enrichment in dendritic spines. Together, these results reveal a novel function of postsynaptic endophilin A1 in spine morphogenesis, stabilization and synaptic function through the regulation of p140Cap.

Keywords: endophilin A1; p140Cap; spine morphogenesis; actin cytoskeleton; synapse formation Cell Research (2015) 25:496-516. doi:10.1038/cr.2015.31; published online 13 March 2015

\section{Introduction}

Dendritic spines are micron-sized postsynaptic structures emanating from neuronal dendrites that receive excitatory synaptic input in the brain. Changes in the shape, size and number of dendritic spines have been linked to synaptic plasticity, which provides structural basis for learning and memory [1]. During development, the shape of dendritic protrusions change from thin, elongated and highly motile filopodium-like structures to more stable stubby spines and mature into mushroom-shape spines with a distinct neck and head $[2,3]$. Abnormal dendritic spine morphology and density has been linked to a num-

Correspondence: Jia-Jia Liu

Tel/Fax: +86-010-64806561

E-mail: jjliu@genetics.ac.cn

Received 4 July 2014; revised 24 November 2014; accepted 12 December 2014; published online 13 March 2015 ber of neuropsychiatric diseases such as schizophrenia and autism spectrum disorders $[4,5]$.

Regulation of actin dynamics by actin-nucleating and -depolymerizing factors plays a key role in dendritic spine morphogenesis and dynamics [6, 7]. Previous studies have established roles of the small GTPase Rif and its effector mDia2, Arp2/3 and ADF/cofilin in the processes of filopodia elongation, spine head expansion and maintenance of spine length and morphology, respectively [8]. However, our understanding of regulatory mechanisms underlying actin-dependent spine maturation and dynamics is still incomplete.

Endophilin A1 is a member of the evolutionarily conserved endophilin A family that is primarily expressed in the nervous system. Endophilin A was first identified as a binding partner for synaptojanin, a component of the endocytic machinery required for synaptic vesicle recycling $[9,10]$. In mammals endophilin $\mathrm{A}$ is encoded by three genes $[9,10]$. Both endophilin A2 and A3 interact 
with Arc/Arg3.1 to regulate endocytic trafficking of AMPA-type glutamate receptors and synaptic plasticity [11, 12]. It was reported recently that although single knockout mice appear normal and fertile, double knockout of endophilin A1 and A2 or knockout of all three endophilins in mouse causes accumulation of clathrin-coated vesicles at the presynaptic site, indicating that they play redundant functions in vesicle uncoating after membrane fission [13]. Although a critical role of endophilin A1 in regulating synaptic vesicle endocytosis at the presynaptic terminal has been well established [14-21], its function at the postsynaptic site remains largely unexplored.

Previously we have found that endophilin A1 interacts with retrolinkin and mediates BDNF-TrkB endocytic trafficking and signaling during dendrite outgrowth [22]. In this study, we used genetic manipulations in combination with biochemical and cell biological approaches to investigate the role of endophilin A1 in dendritic spines. To avoid compensatory effects or functional redundancy of endophilin A2 and/or A3 when endophilin A1 activity is abolished, we depleted endophilin A1 by short hairpin RNA (shRNA)-mediated knockdown in primary cultured rat hippocampal and cortical neurons. We show that endophilin A1 interacts with p140Cap and that this interaction is required for spine morphogenesis and stabilization. Moreover, inhibition of the endophilin A1-p140Cap pathway causes a decrease in the levels of cortactin, an actin remodeling factor and p140Cap interaction partner, in dendritic spines. We propose that by modulating the distribution of cortactin in spines, endophilin A1 cooperates with p140Cap to regulate rearrangements of the actin cytoskeleton during spine formation and maturation, thereby promoting synapse formation.

\section{Results}

\section{Endophilin A1 localizes in dendritic spines}

It has been reported that endophilin A1 is mainly expressed at the presynaptic site [11] and plays a critical role in synaptic vesicle recycling via regulation of clathrin-mediated endocytosis [14-21]. However, our previous study has demonstrated that endophilin A1 interacts with retrolinkin in dendrites of mammalian central nervous system neurons and mediates the endocytic trafficking and signaling of the BDNF-TrkB ligand-receptor complex during dendrite outgrowth [22]. To further explore its function(s) in dendrites, we first examined its expression levels in mouse brain at different developmental stages. Immunoblotting analysis revealed that expression of both endophilin A1 and endophilin B1, another member of the endophilin family, increased after birth and maintained at high levels in adult mice. In contrast, no increase in expression levels of endophilin A2 and A3 was detected throughout development (Figure 1A). These data suggest that endophilin A1 functions at the late stages of neurodevelopment, such as synapse maturation and neural circuitry formation. Next, to determine the subcellular distribution of endophilin A1 in dendrites, we performed immunofluorescence staining with antibodies to endophilin A1 in cultured hippocampal neurons transfected with red fluorescent protein (DsRed)-expressing construct. Confocal microscopy and structured illumination microscopy analyses indicated that the endophilin A1 clusters distributed to both dendritic shafts and spines (Figure 1B and 1C) and some clusters were in close proximity to or partially colocalized with the postsynaptic density (PSD) structure labeled with antibodies to PSD95 (Figure 1C, Supplementary information, Figure S1A and S1C, and Movie S1). Consistently, subcellular fractionation assays revealed that a significant amount of endophilin A1 was distributed in the PSD fraction (Figure 1D). Moreover, in agreement with previous reports [11], immunogold labeling of ultrathin sections with antibodies to endophilin A1 detected its signals in the postsynaptic spines of mouse hippocampus (Figure $1 \mathrm{E})$. Live imaging of hippocampal neurons also revealed distribution of a endophilin A1-GFP fusion protein (EENA1-LentiGFP) in both axon boutons and dendritic protrusions, with fluorescent signals in both tips of filopodia and mushroom-shaped spines (Figure 1F). These data prompted us to investigate whether endophilin A1 also functions in the postsynaptic compartment.

Endophilin A1 interacts with p140Cap via its SH3 domain in dendritic spines

To investigate function(s) of endophilin A1 in the postsynaptic compartment, we performed GST pull-down assay from mouse brain lysates using recombinant GSTtagged endophilin A1 to search for its interaction partners other than synaptojanin and retrolinkin. Mass spectrometry analysis of bound proteins showed that endophilin A1 not only interacted with synaptojanin and dynamin, components of the endocytic machinery $[10,13,16]$, but also with p130Cas-associated protein (p140Cap, also known as SNAP-25-interacting protein), a protein regulating cytoskeleton reorganization [23, 24] (Supplementary information, Figure S1D). Co-immunoprecipitation (CoIP) assay from HEK293 cells co-expressing Flag-tagged endophilins and Myc-tagged p140Cap revealed that both endophilin A1 and A3 interacted with p140Cap, whereas endophilin A2 and B1 weakly or hardly associated with p140Cap (Figure 2A). Similar results were obtained by Co-IP assay using mouse brain lysates (Figure 2B).

All members of the endophilin family contain a BAR 
A

E14 E16 E18 P0 P7 P21 Adult

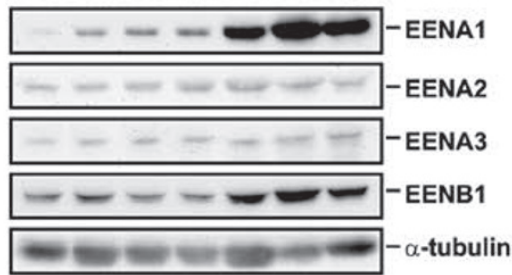

B

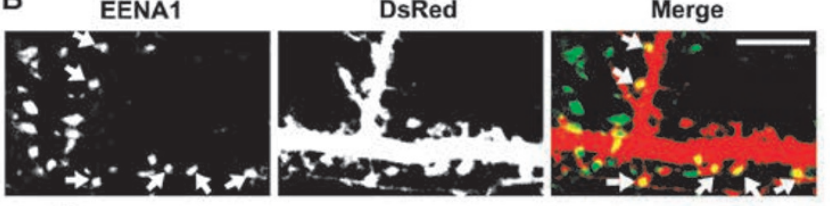

D S1 S2 P2 S3 P3 S4 PSD



E

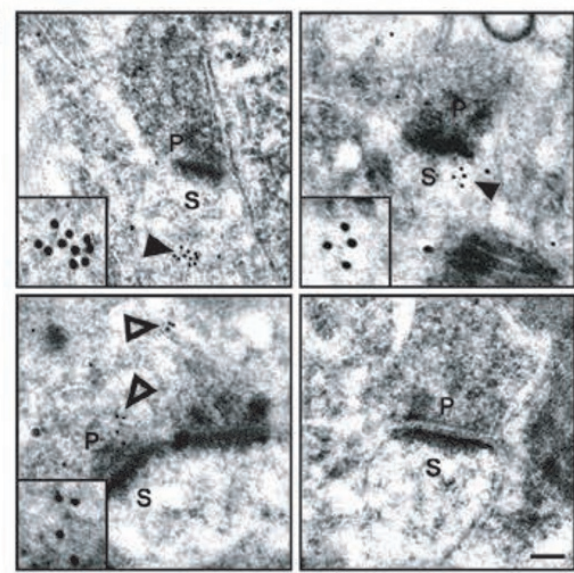

C



$\mathbf{F}$
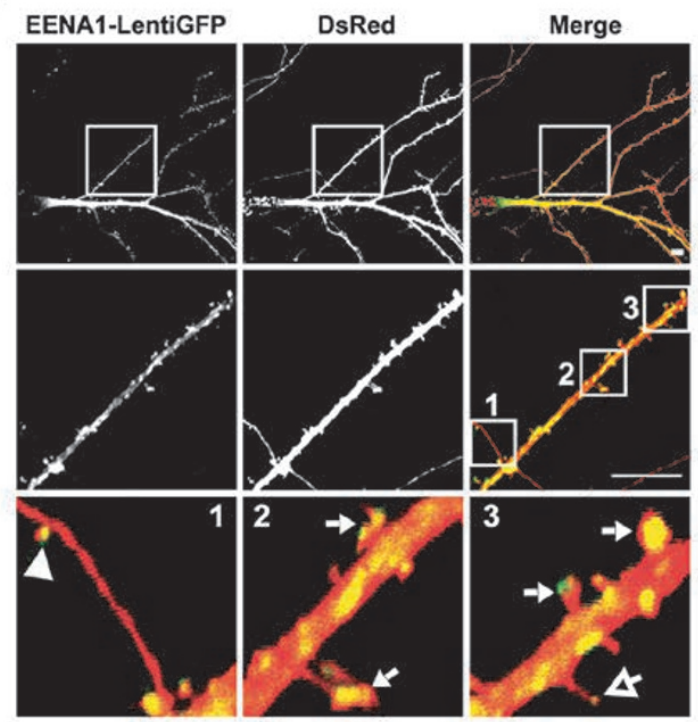

3



Figure 1 Endophilin A1 localizes in dendritic shafts and spines in mature neurons. (A) Expression patterns of endophilin family members during brain development. Western blot analyses of mouse brain lysates were performed with antibodies to endophilin (EEN) A1, A2, A3 and B1. E, embryonic; P, postnatal. (B) DIV21 hippocampal neurons expressing the red fluorescent protein DsRed as volume marker were immunostained with antibodies to endophilin A1. Shown are representative confocal microscopical images. Arrows indicate EENA1 localization in spines. Scale bar, $5 \mu \mathrm{m}$. (C) DIV21 hippocampal neurons expressing DsRed were immunostained with antibodies to endophilin A1 (green) and PSD95 (blue). Shown are representative z stack maximum projection images captured by structured illumination microscopy (SIM). Lower panels are insets of boxed areas in the upper panel. Dendrites and spines are outlined in white. Scale bar, $5 \mu \mathrm{m}$. (D) Mouse brain lysates were fractionated by differential centrifugation and subcellular fractions were analyzed by immunoblotting with antibodies to endophilins and other neuronal proteins. S1, homogenates; S2, supernatant after P2 precipitation; P2, crude synaptosomes; S3, cytosol; P3, light membranes; S4, supernatant after postsynaptic density (PSD) precipitation. SYP, synaptophysin. (E) Immunogold labeling of mouse hippocampal ultrasections with antibodies to endophilin A1. Open and filled arrowheads indicate endophilin A1 signals in presynapses and postsynaptic spines, respectively. S, spine; $\mathrm{P}$, presynapse. The bottom right panel is negative control with secondary antibodies only. Scale bar, $100 \mathrm{~nm}$. (F) Confocal images of DIV21 hippocampal neurons co-expressing EENA1-green fluorescent protein (GFP) fusion (EENA1-LentiGFP) and DsRed. Lower panels are insets of boxed area(s) in the upper panels. Arrowhead, axon bouton; filled arrows, mushroom-like spines; open arrow, filopodial tip. Scale bar, $10 \mu \mathrm{m}$. 
A

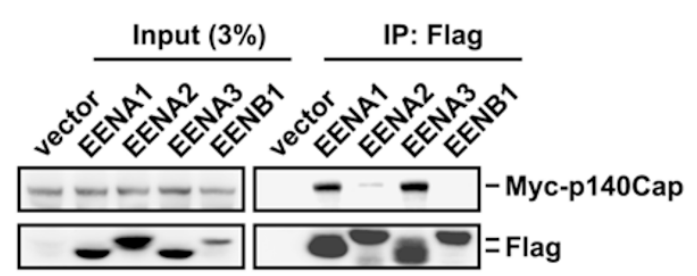

B



$\mathrm{E}$

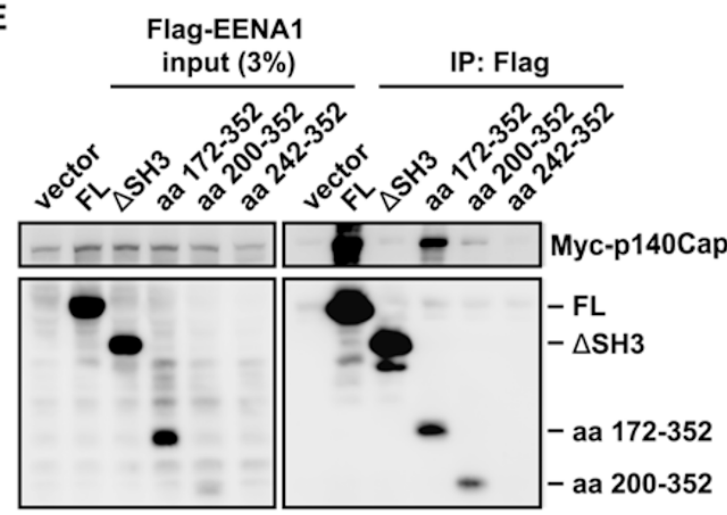

G
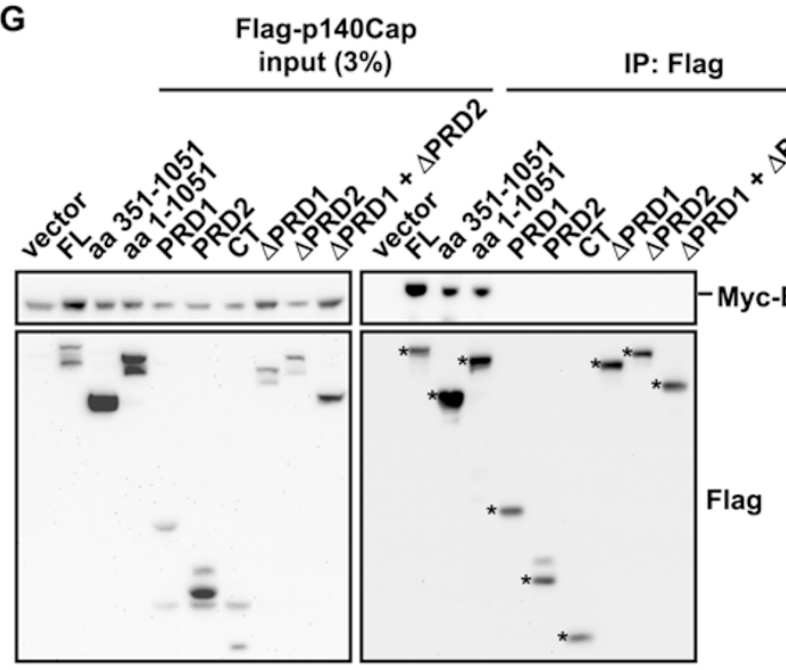

domain and an SH3 domain that frequently interacts with proline-rich domains (PRDs) of other proteins (Figure 2C) [25]. We then determined which domain in endophilin A1 interacts with p140Cap. Although a fragment
C

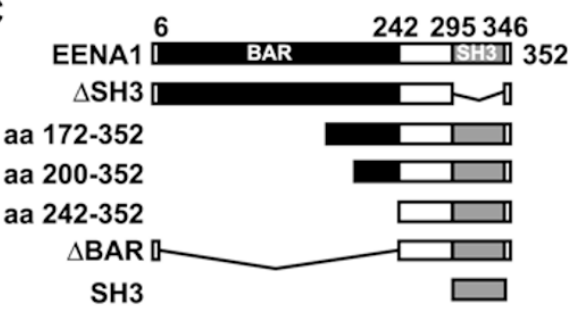

D
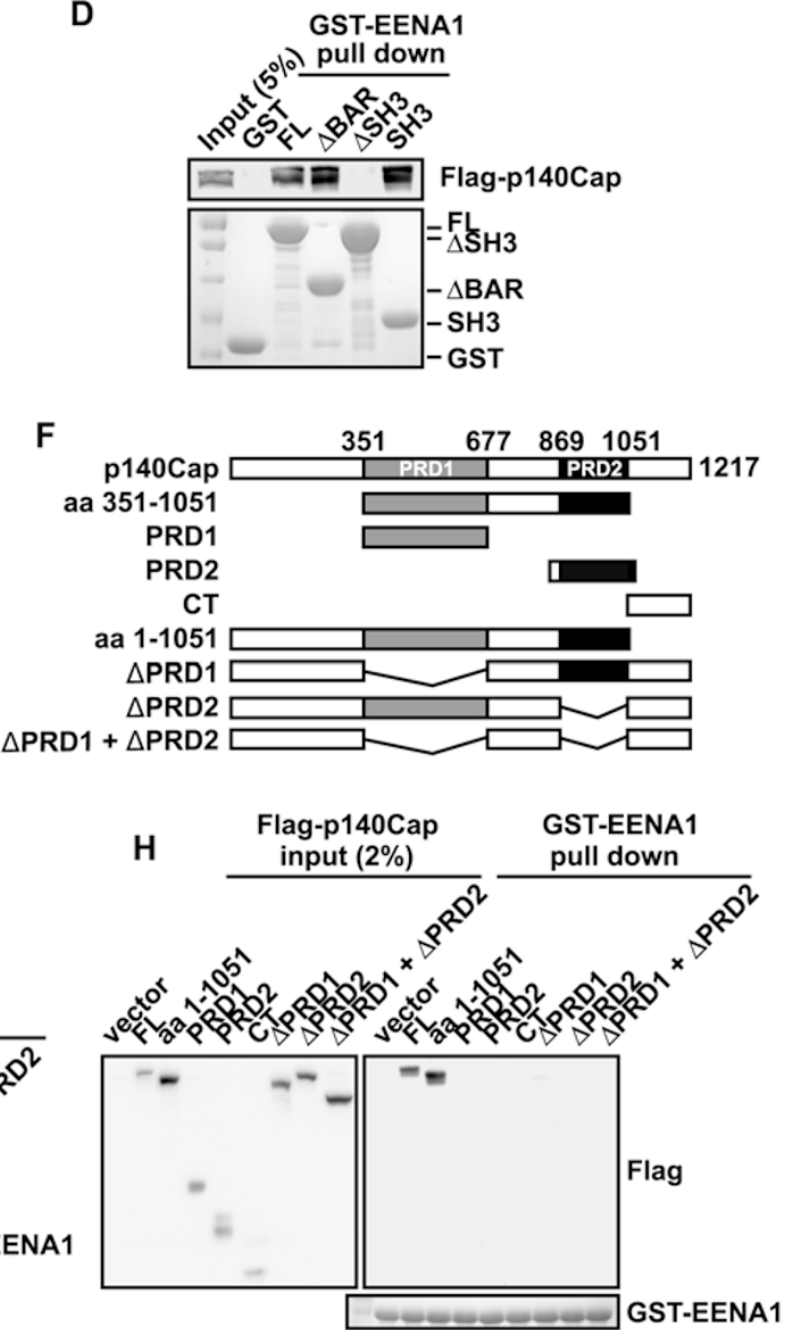

\section{I}
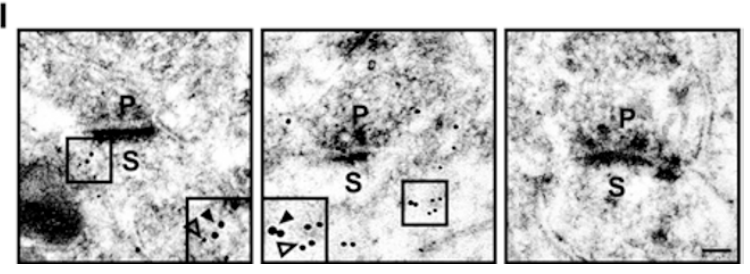

of endophilin A1 lacking the BAR domain (aa 6-242, $\triangle B A R$ ) did not express in HEK293 cells, all Flag-tagged endophilin A1 fragments (aa 172-352 and aa 200-352) containing the SH3 domain (aa 295-346) co-immuno- 
Figure 2 Endophilin A1 binds to p140Cap via its SH3 domain. (A) HEK293 cells cotransfected with constructs expressing Myc-tagged p140Cap and Flag-tagged endophilin for $48 \mathrm{~h}$ were lysed for immunoprecipitation with immobilized Flag antibody. Input and bound proteins were analyzed by immunoblotting with antibodies against Myc and Flag. (B) Cultured cortical neurons were lysed for immunoprecipitation with antibodies to endophilins. Input and bound proteins were analyzed by immunoblotting with antibodies to endophilins and p140Cap. (C) Schematic representation of endophilin A1 domain structure and diagrams of endophilin A1 fragments used for interaction site mapping. (D) GST-tagged endophilin A1 fragments conjugated to glutathione-Sepharose were incubated with lysates of HEK293 cells overexpressing Flag-tagged p140Cap. Input and bound proteins were analyzed by immunoblotting with antibodies against Flag. Bottom panel: coomassie blue-stained SDSPAGE gel showing GST fusion proteins. FL, full length. (E) HEK293 cells cotransfected with constructs encoding Myc-tagged p140Cap and Flag-tagged endophilin A1 fragments were lysed for immunoprecipitaion with immobilized Flag antibody. Input and bound proteins were analyzed by immunoblotting with antibodies against Myc and Flag. (F) Schematic representation of p140Cap domain structure and diagrams of p140Cap fragments used for mapping. (G) HEK293 cells cotransfected with constructs encoding Myc-tagged endophilin A1 and Flag-tagged p140Cap fragments were lysed for immunoprecipitaion with immobilized Flag antibody. Input and bound proteins were analyzed by immunoblotting with antibodies against Myc and Flag. Asterisks indicate the bands of Flag-tagged p140Cap fragments. (H) GST-tagged endophilin A1 conjugated to glutathione-Sepharose was incubated with the lysates from HEK293 cells overexpressing Flag-tagged p140Cap fragments. Input and bound proteins were analyzed by immunoblotting with antibodies against Flag. Bottom panel: coomassie blue-stained SDS-PAGE gel showing GST-EENA1. (I) Representative images of double immunogold labeling of mouse hippocamal ultrathin sections with antibodies against endophilin A1 (12 nm, open arrowheads) and p140Cap (18 nm, filled arrowheads). Right panel is the negative control with secondary antibodies only. Scale bar, $100 \mathrm{~nm}$.

precipitated with Myc-p140Cap in transiently transfected HEK293 cells, whereas deletion of the SH3 domain $(\triangle \mathrm{SH} 3)$ abolished binding of endophilin A1 to p140Cap (Figure 2E and Supplementary information, Figure S1F). Consistently, GST pull-down with endophilin A1 fused to GST showed that deletion of the SH3 ( $\triangle \mathrm{SH} 3)$, not the BAR domain ( $\triangle \mathrm{BAR}$ ), abolished their interaction and that the SH3 domain fragment could pull down p140Cap (Figure 2D and Supplementary information, Figure $\mathrm{S} 1 \mathrm{~F})$. Together, these data indicate that the SH3 domain of endophilin A1 binds to p140Cap.

Next we determined the endophilin A1 interaction site in p140Cap by Co-IP and GST pull-down assays. Co-IP from HEK293 cells indicated that p140Cap full length and fragments (aa 1-1 051 and aa 351-1 051) containing both PRD1 and PRD2 could bind to endophilin A1, whereas PRD1 (aa 351-677), PRD2 (aa 841-1 051), the carboxyl terminus (aa $1052-1217, \mathrm{CT}$ ), $\triangle \mathrm{PRD} 1$ (deletion of aa 351-677), $\triangle \mathrm{PRD} 2$ (deletion of aa 869-1 051) and $\triangle \mathrm{PRD} 1+\triangle \mathrm{PRD} 2$ (deletion of aa 351-677 and aa 869-1 051) could not (Figure 2F, 2G and Supplementary information, Figure S1G). Consistently, GST pull-down assay with endophilin A1 fused to GST also indicates that both PRD1 and PRD2 are required for the interaction of p140Cap with endophilin A1 (Figure 2H).

To further confirm the interaction, we performed double immunofluorescence staining of cultured hippocampal neurons after 21 days in vitro (DIV) with antibodies to endophilin A1 and p140Cap. Confocal microscopy analysis showed that endophilin A1 and p140Cap co-localized in dendritic spines in cultured hippocampal neurons (Supplementary information, Figure S1B and S1C).
Moreover, their colocalization in dendritic spines was verified by immunoelectron microscopy (immunoEM) analysis of mouse brain ultrathin sections (Figure 2I). Taken together, these results indicate that endophilin A1 interacts with p140Cap via its SH3 domain in dendritic spines.

Endophilin A1 in dendritic spines specifically regulates spine morphogenesis, synapse formation and function

It has been reported that p140Cap is tightly associated with cytoskeleton [23] and is enriched in the PSD fraction [26] (Figure 1D) and that p140Cap silencing causes a decrease in the number of spines and an increase in the number of filopodia [26]. To determine whether endophilin A1 also regulates spine morphology, we depleted endophilin A1 by shRNA-mediated RNA interference (RNAi) in cultured hippocampal neurons on DIV1617 , and monitored dendritic spine morphology by the fluorescence of co-expressed DsRed or GFP on DIV21. Immunoblotting and immunofluorescence staining analyses indicated that shRNA efficiently knocked down the expression of exogenous proteins in HEK293 cells as well as endogenous proteins in neurons (Supplementary information, Figure S2A-S2G).

Confocal microscopy analysis showed that silencing of endophilin A1 led to a decrease in the numbers of total protrusions and spines but an increase in the number of filopodia (Figure 3A and 3B). In contrast, knockdown of other endophilins had no obvious effect on spine morphology (Figure 3A and 3B). Further, defects in spine morphogenesis were rescued by coexpression of RNAi-resistant endophilin A1 but not by coexpression of 
endophilin A2 or A3 (Figure 3C, 3D and Supplementary information, Figure S2A).

A majority of excitatory synapses are formed at dendritic spines and changes in spine morphology are associated with differences at synaptic level. To determine role(s) of endophilins in excitatory synapse formation, we examined PSD95 puncta in dendrites which indicate the excitatory postsynaptic sites by immunostaining of dissociated hippocampal neurons after shRNA transfection. Confocal microscopy analysis indicated that silencing of endophilin A1 caused a decrease in the intensity of PSD95 in dendrites (Figure 3C and Supplementary
A



EENA1-ShRNA\#2

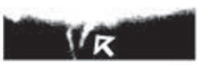

EENA2-shRNA torat?

EENA3-ShRNA $6+\mathrm{y}_{2}$



EENB1-shRNA

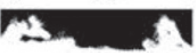

E

Ctrl-shRNA

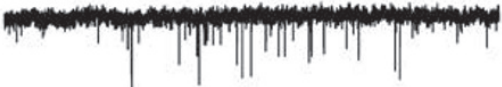

EENA1-ShRNA

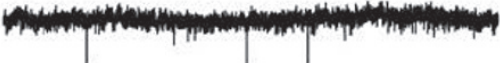

EENA1-shRNA + EENA1*

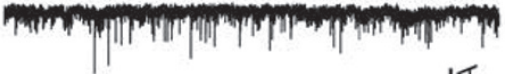<smiles>C1=C[AsH2]C1</smiles>

$\mathbf{F}$

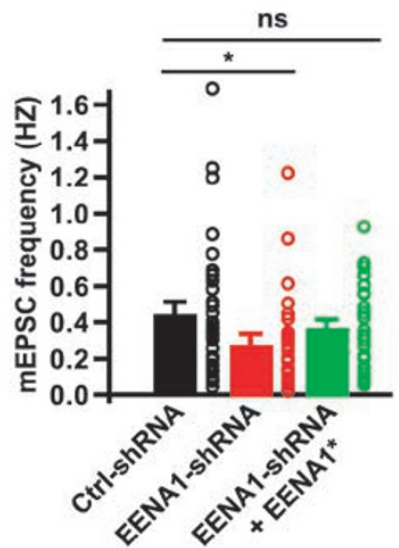

G
B

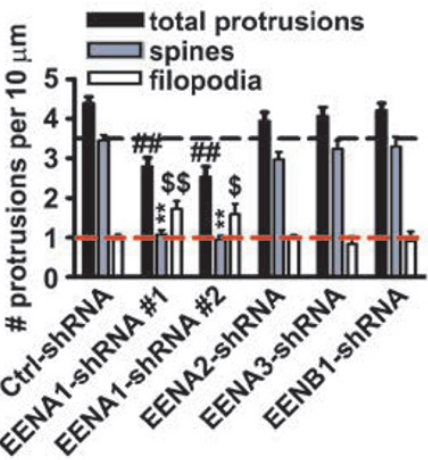

C
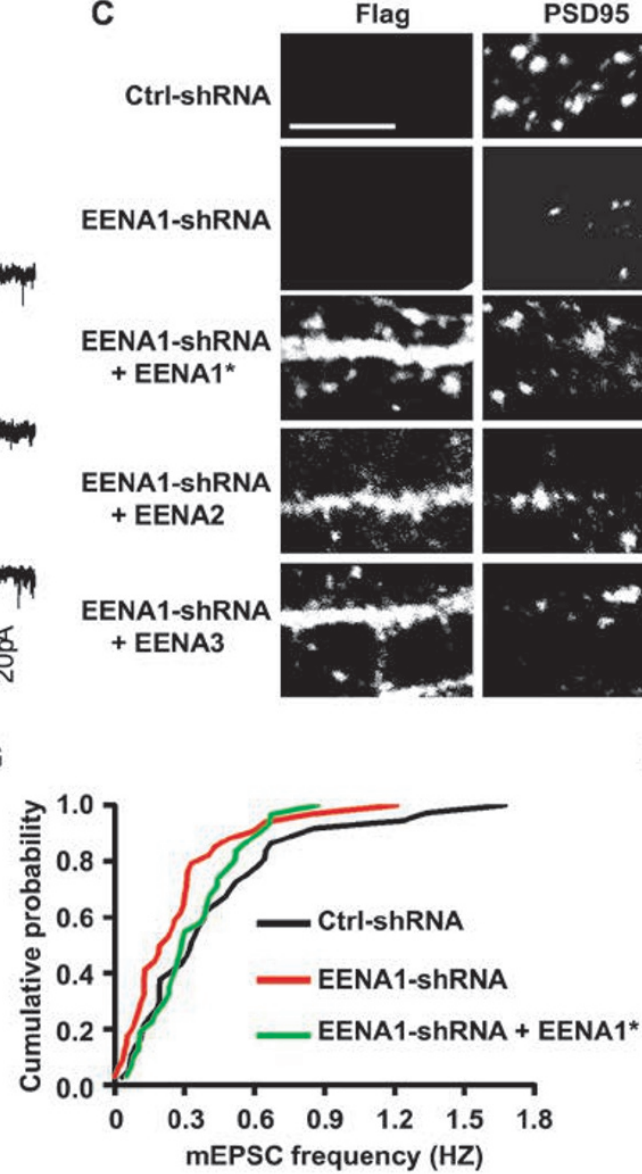
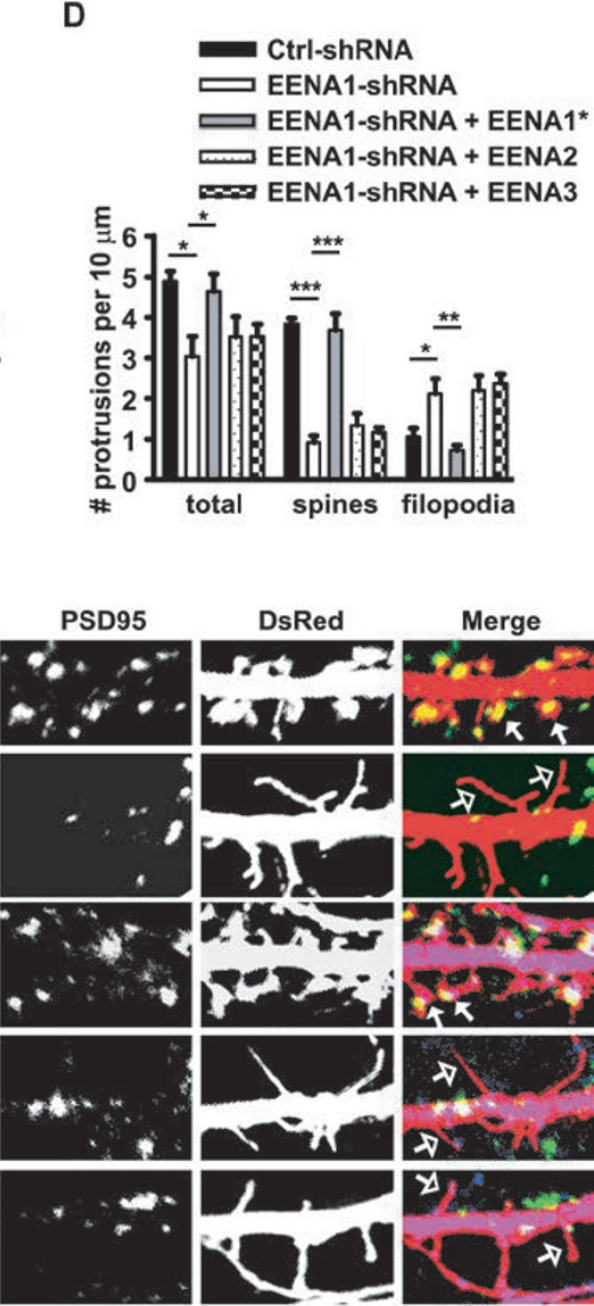

H

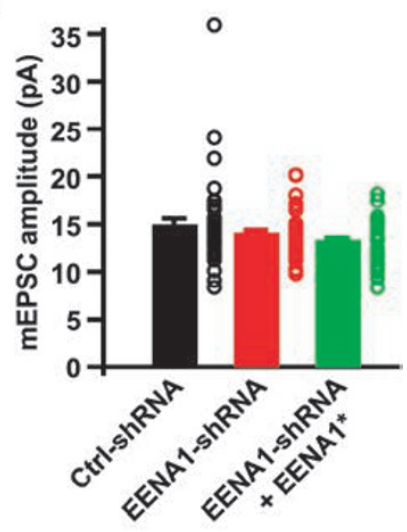


information, Figure $\mathrm{S} 2 \mathrm{H}$ ) and led to reduced number and area in PSD95 puncta in dendrites (Figure 3C, Supplementary information, Figure S2I and S2J), especially the number of larger puncta (Supplementary information, Figure S2K), indicating that the formation of postsynaptic sites is hampered in endophilin A1 knockdown neurons and the remaining postsynaptic sites are smaller than control. Consistently, defects in synapse formation were rescued by coexpression of RNAi-resistant endophilin A1 but not by coexpression of endophilin A2 or A3 (Figure $3 \mathrm{C}$ and Supplementary information, Figure S2H$\mathrm{S} 2 \mathrm{~K})$. These data indicate that endophilin A1 specifically regulates spine morphogenesis and synapse formation.

To determine whether the functionality of endophilin A1-silenced neurons is changed, we performed electrophysiological analysis and found that the frequency but not the amplitude of miniature excitatory postsynaptic currents (mEPSCs) was reduced compared with control shRNA-expressing neurons (Figure 3E-3H, Supplementary information, Figure S2L), indicating a decrease in the number of functional synapses. This phenotype was rescued by overexpression of RNAi-resistant endophilin A1 (Figure 3E-3H, Supplementary information, Figure S2L).

Endophilin Al in dendritic spines regulates spine morphogenesis and synapse formation through binding to p140Cap

Knockdown of either p140Cap (see Supplementary information, Figure S3A-S3D for knock-down efficien- cy) or endophilin A1 resulted in similar phenotypes in spine morphology, characterized by an increased number in filopodia and a decreased number in spines, and filopodia growing from the existing spine head were also observed (Supplementary information, Figure S3E and S3F). Previously it was found that in addition to spine phenotype, p140Cap silencing also causes a reduction in the frequency but not amplitude of mEPSCs [27], which is similar to the phenotypes caused by endophilin A1 silencing. That downregulation of either endophilin A1 or p140Cap causes similar phenotypes in spine morphology and neuronal function led us to speculate that these two proteins act in the same pathway to regulate spine morphogenesis.

To determine whether endophilin A1 regulates spine morphology and synapse formation through binding to p140Cap, we cotransfected cultured hippocampal neurons with constructs expressing endophilin A1-shRNA and RNAi-resistant endophilin A1, endophilin A1- $\Delta \mathrm{SH} 3$, or p140Cap. Overexpression of the p140Cap binding-deficient $\triangle \mathrm{SH} 3$ mutant could not rescue the defects in spine morphology caused by endophilin A1 knockdown, whereas overexpression of p140Cap could fully rescue the defects (Figure 4A and 4B). We also examined synapse formation by immunostaining of PSD95 and found that coexpression of the $\Delta \mathrm{SH} 3$ mutant could not rescue the defects in the formation of post-synaptic sites caused by endophilin A1 knockdown, whereas overexpression of p140Cap could fully rescue the defects (Figure 4A, $4 \mathrm{C}-4 \mathrm{~F})$. We further confirmed the regulation of synapse

Figure 3 Endophilin A1 is required for dendritic spine morphogenesis and synaptic function. (A) Cultured hippocampal neurons transfected with shRNA constructs coexpressing shRNA and DsRed at DIV16-17 followed by immunostaining with antibodies to DsRed at DIV21. Shown are representative confocal images. Filled arrows, spines; Open arrows, filopodia. Ctrl: non-targeting shRNA. Scale bar, $5 \mu \mathrm{m}$. (B) Quantification of dendritic protrusion density of transfected neurons in A (number of cells analyzed, Ctrl-shRNA: 31, EENA1-shRNA \#1: 20, EENA1-shRNA \#2: 15, EENA2-shRNA: 18, EENA3-shRNA: 17, EENB1-shRNA: 15). In all, more than 600 protrusions were measured for each group. All values are shown as mean \pm SEM. Statistical test: ${ }^{\#} P<0.01$ (total protrusions), ${ }^{* *} P<0.01$ (spines), ${ }^{\$ \$} P<0.01,{ }^{\$} P<0.05$ (filopodia); one-way ANOVA followed by Dunnett's multiple-comparison post hoc tests. (C) Representative confocal images of cultured hippocampal neurons transfected with shRNA constructs or cotransfected with constructs encoding shRNA and RNAi-resistant Flag-tagged EENA1 (indicated by asterisk), Flag-tagged EENA2, or Flag-tagged EENA3 at DIV16-17 followed by immunostaining with antibodies against PSD95 (green), Flag (blue) and DsRed at DIV21. Filled arrows, spines; open arrows, filopodia. Scale bar, $5 \mu \mathrm{m}$. (D) Quantitative analysis of dendritic spine protrusion density in C (number of cells analyzed, Ctrl-shRNA: 18, EENA1-shRNA: 15, EENA1-shRNA + EENA1*: 18, EENA1-shRNA + EENA2: 18, EENA1-shRNA + EENA3: 19). More than 550 protrusions were analyzed for each group. All values are shown as mean \pm SEM. Statistical test: ${ }^{* \star *} P<0.001,{ }^{* *} P<0.01,{ }^{\star} P<0.05$; one-way ANOVA followed by Newman-Keuls multiple comparison post hoc tests. (E) Cultured hippocampal neurons were transfected with shRNA constructs or cotransfected with constructs encoding shRNA and RNAi-resistant mCherry-tagged EENA1 (indicated by asterisk) at DIV9-10 followed by mEPSC recording at DIV14-16. Shown are representative traces of mEPSC recordings. (F) Quantitative analysis of mEPSC frequency. Ctrl-shRNA: $n=4$ independent experiments, 37 neurons; EENA1-shRNA: $n=4,34$ neurons; EENA1-shRNA + EENA1*: $n=4,33$ neurons. Statistical test: * $P<0.05$; one-way ANOVA followed by Dunnett's multiple-comparison post hoc tests. ns, not significant. (G) Cumulative distribution of mEPSC frequency. Statistical test: $P=0.012$ for EENA1-shRNA versus Ctrl-shRNA, two-sample Kolmogorov-Smirnov test. (H) Quantitative analysis of mEPSC amplitude. 




formation by detecting synaptophysin, a presynaptic protein, on dendrites of transfected neurons. Consistently, overexpression of $\triangle \mathrm{SH} 3$ mutant had no rescue effects on either the fewer synaptophysin puncta on dendrites or the lower colocalization of synaptophysin with dendrites caused by endophilin A1 knockdown, compared with 
Figure 4 Endophilin A1 regulates spine morphogenesis and synapse formation through its interaction with p140Cap. (A) Cultured hippocampal neurons were transfected with shRNA constructs or cotransfected with constructs encoding EENA1-shRNA and RNAi-resistant Flag-tagged endophilin A1, RNAi-resistant Flag-tagged endophilin A1 lacking SH3 domain ( $\triangle \mathrm{SH}$; indicated by asterisk), Flag-tagged p140Cap, or constructs encoding p140Cap-shRNA and Flag-tagged endophilin A1 at DIV16-17 followed by immunostaining with antibodies to PSD95 (green), Flag (blue) and DsRed at DIV21. Shown are representative confocal images. Filled arrows, spines; open arrows, filopodia. Scale bar, $5 \mu \mathrm{m}$. (B) Quantification of dendritic protrusion density of transfected neurons in $\mathbf{A}$ (number of cells analyzed, Ctrl-shRNA: 15, EENA1-shRNA: 15, EENA1-shRNA + EENA1*: 15, EENA1-shRNA + $\triangle$ SH3*: 18, EENA1-shRNA + p140Cap FL: 15, p140Cap-shRNA: 15, p140Cap-shRNA + EENA1: 18). More than 500 protrusions were analyzed for each group. All values are shown as mean \pm SEM. Statistical test: ${ }^{* *} P<0.001,{ }^{* *} P<0.01,{ }^{*} P<0.05$; one-way ANOVA followed by Newman-Keuls multiple comparison post hoc tests. (C-E) Quantification of PSD95 intensity (C), puncta number (D) and puncta area (E) in dendrites of transfected neurons in A (number of cells analyzed, Ctrl-shRNA: 23, EENA1-shRNA: 25, EENA1-shRNA + EENA1*: 24, EENA1-shRNA + $\triangle$ SH3*: 22, EENA1-shRNA + p140Cap FL: 18, p140Cap-shRNA: 18, p140Cap-shRNA + EENA1: 28. number of puncta analyzed, Ctrl-shRNA: 990, EENA1-shRNA: 578, EENA1-shRNA + EENA1*: 1489, EENA1-shRNA + $\triangle$ SH3*: 449, EENA1-shRNA + p140Cap FL: 909, p140Cap-shRNA: 397, p140Cap-shRNA + EENA1: 432). More than $1000 \mu$ of dendrite length was analyzed for each group. All values are shown as mean \pm SEM. Statistical test: ${ }^{* *} P<0.001$; one-way ANOVA followed by Dunnett's multiple-comparison post hoc tests. (F) Cumulative distribution of PSD95 puncta area. (G) Cultured hippocampal neurons were transfected with shRNA constructs or cotransfected with constructs encoding EENA1-shRNA and RNAi-resistant Flag-tagged endophilin A1, RNAi-resistant Flag-tagged endophilin A1 lacking SH3 domain ( $\triangle \mathrm{SH} 3$; indicated by asterisk), Flag-tagged p140Cap, or constructs encoding p140Cap-shRNA and Flag-tagged endophilin A1 at DIV16-17 followed by immunostaining with antibodies to synaptophysin (SYP, green), Flag (blue) and DsRed at DIV21. Shown are representative confocal images. Filled arrows, spines; Open arrows, filopodia. Scale bar, $5 \mu \mathrm{m}$. (H, I) Quantitative analysis of the SYP puncta number along dendrites $(\mathbf{H})$ or percentage of dendritic area colocalizing with SYP (I) (number of cells analyzed, Ctrl-shRNA: 16, EENA1-shRNA: 18, EENA1-shRNA + EENA1*: 15, EENA1-shRNA + $\triangle$ SH3*: 15, EENA1-shRNA + p140Cap FL: 16, p140Cap-shRNA: 13, p140Cap-shRNA + EENA1: 15). More than $600 \mu \mathrm{m}$ of dendrite length was analyzed for each group. All values are shown as mean \pm SEM. Statistical test: ${ }^{* * *} P<0.001$; one-way ANOVA followed by Dunnett's multiple-comparison post hoc tests.

control neurons, whereas overexpression of p140Cap could fully rescue the defects (Figure 4G-4I). Taken together, these results indicate that the interaction between endophilin A1 and p140Cap is required for the regulatory function of endophilin A1 in spine morphogenesis and synapse formation.

To determine whether p140Cap acts downstream of endophilin A1 in spine morphogenesis and synapse formation, we investigated the rescue effects of endophilin A1 on spine morphogenesis and synapse formation in p140Cap-silenced neurons. Although overexpression of endophilin A1 had a small but significant rescue effect on the numbers of spines and total protrusions induced by p140Cap knockdown (Figure 4A and 4B), overexpression of endophilin A1 had no rescue effects on synapse formation of p140Cap-silenced neurons (Figure 4A, 4C$4 \mathrm{I}$ ), suggesting that $\mathrm{p} 140 \mathrm{Cap}$ is an important downstream effector of endophilin A1. The small rescue effects on spine morphology may be attributable to the presence of additional effectors downstream of endophilin A1 to regulate spine morphogenesis. Collectively, these results indicate that the endophilin A1-p140Cap pathway regulates spine morphogenesis which is crucial for excitatory synapse formation, and that p140Cap functions downstream of endophilin A1.

To further verify that the interaction between endophi- lin A1 and p140Cap is required for spine morphogenesis, we mutated six evolutionarily conserved residues (Y299, F301, E308, W327, P340, Y343) in the SH3 domain of endophilin A1 predicted to be important for binding to PRD domains (NCBI/CDD, cd11803) to alanine respectively and tested their binding ability by Co-IP assay in HEK293 cells. Mutation of P340 to alanine enhanced the interaction between endophilin A1 and p140Cap (Supplementary information, Figure S4A), but this mutant failed to express in cultured neurons. Nevertheless, compared with wild-type (WT) endophilin A1, mutation of Y299 or Y343 to alanine significantly weakened or completely abolished its interaction with p140Cap, whereas mutation of other residues to alanine had no obvious effect (Supplementary information, Figure S4A). Accordingly, Y299A and Y343A failed to rescue the defects in spine morphology in endophilin A1-silenced neurons (Supplementary information, Figure S4B and S4C). These results are consistent with the notion that endophilin A1 regulates spine morphorgenesis through binding to p140Cap.

The BAR domain of endophilin A1 is capable of membrane sensing, membrane binding and bending [28]. Although the $\triangle B A R$ fragment of endophilin A1 did not express in mammalian cells, to determine whether BAR domain-mediated membrane association of endophilin A1 is also required for spine morphogenesis, we 
cotransfected cultured hippocampal neurons with constructs expressing endophilin A1-shRNA and endophilin A1 mutant (triple point mutation KKK-EEE or BAR$\Delta \mathrm{H} 1 \mathrm{I})$ which is less effective in membrane binding and tubulation [28]. Overexpression of endophilin A1 KKKEEE or BAR- $\triangle H 1 I$ had a small but significant rescue effect on spine morphology, but their rescue effects were obviously hampered, compared with endophilin A1 WT

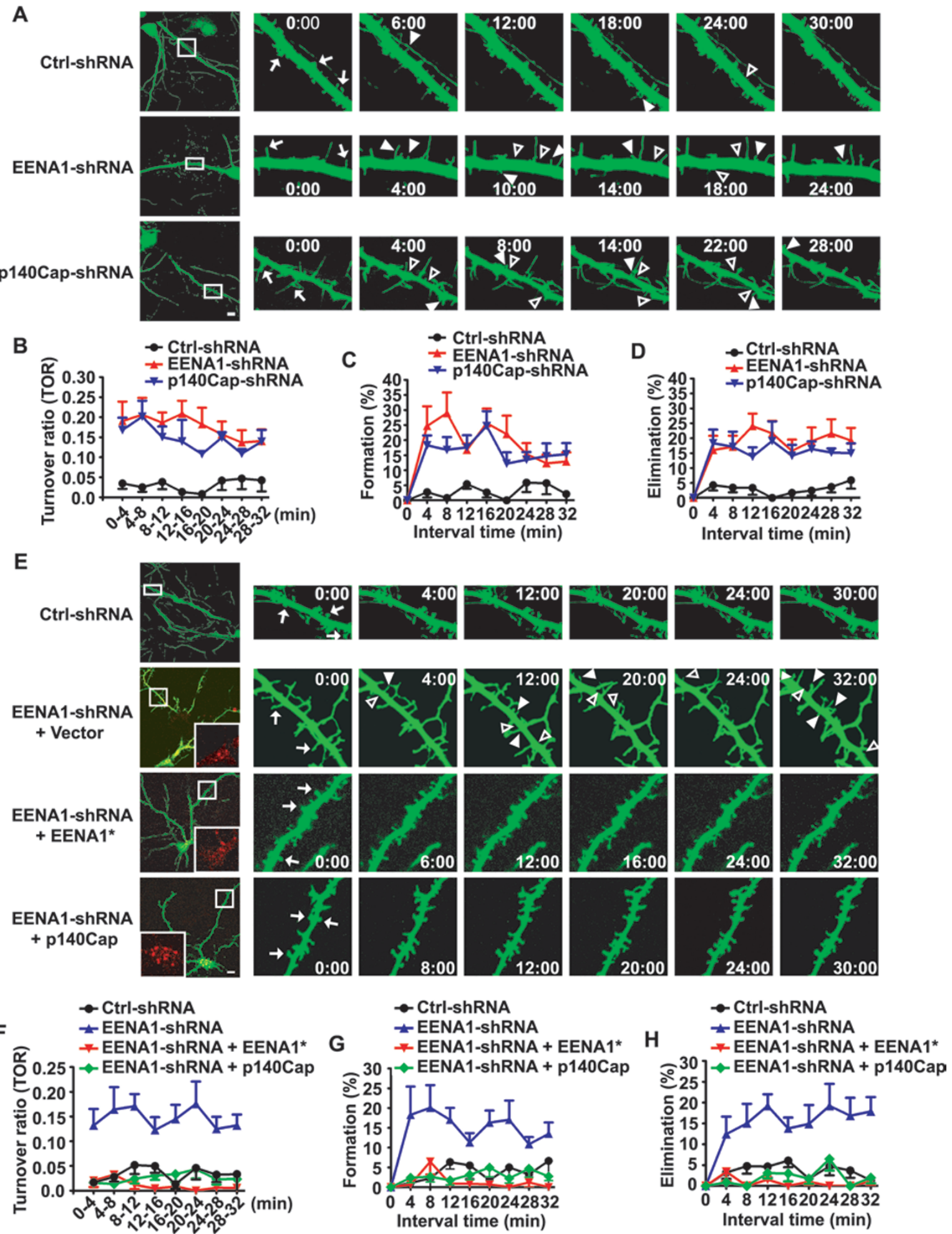


(Supplementary information, Figure S4D and S4E). Taken together, these results indicate that the regulation of spine morphogenesis by endophilin A1 requires not only binding to p140Cap but also its membrane association.

\section{Endophilin A1 and p140Cap promote dendritic spine sta- bilization}

To explore the mechanistic role of endophilin A1 and p140Cap in spine morphogenesis, we knocked down endophilin A1 or p140Cap in cultured hippocampal neurons and monitored dendritic protrusion dynamics for $~$ 30 min by live imaging. In Ctrl-shRNA-transfected neurons, dendrites were predominantly covered with spines which were largely stable with few appearances of new protrusions or loss of existing protrusions (Figure 5A). In contrast, dendrites of endophilin A1 shRNA-transfected neurons were mainly covered with filopodia which were highly dynamic with rapid addition and loss, and the dendrites had the ability to form spines and filopodia (Figure 5A). The p140Cap-silenced neurons showed similar phenotypes to endophilin A1-silenced ones (Figure 5A). Quantitative analysis revealed that knockdown of endophilin A1 or p140Cap caused the higher turnover ratio (TOR) of protrusions $(>15 \%$ in EENA1-shRNA neurons, $>10 \%$ in p140Cap-shRNA neurons versus $<$ $5 \%$ in Ctrl-shRNA neurons; Figure 5B) and resulted in the increased percentage of protrusion formation and elimination, compared with control neurons (Figure 5C and 5D). Furthermore, the phenotypes in endophilin A1-silenced neurons were fully rescued by coexpression of RNAi-resistant endophilin A1 or p140Cap (Figure $5 \mathrm{E}-5 \mathrm{H})$. Together, these results indicate that the endophilin A1-p140Cap pathway promotes the stabilization of dendritic spines.
Endophilin A1 regulates actin cytoskeleton reorganization in spine morphogenesis and synaptic function through p140Cap and cortactin

It is well established that spines contain extremely high levels of actin and the actin cytoskeleton plays a pivotal role in the dynamics and morphology of dendritic spines. It was also reported that modulation of actin dynamics by regulatory proteins including WAVE1, Arp2/3 and cortactin drives the morphological changes in dendritic spines that are associated with alteration in synaptic strength [29]. Since p140Cap plays an important function in actin reorganization in dendritic spines, we reasoned that endophilin A1 might modulate the actin cytoskeleton via its interaction with p140Cap. To determine whether endophilin A1 associates with the actin cytoskeleton, we treated HeLa cells expressing GFP-endophilin A1 with Triton X-100 to extract cytosolic proteins and found that while most of the fluorescent signal was lost, some GFP-endophilin A1 remained as dispersedly distributed small granular structures (Figure 6A and $6 \mathrm{~B})$. In contrast, the number of fluorescent puncta was significantly decreased when endophilin A1 was deleted of the SH3 domain (GFP- $\triangle \mathrm{SH} 3$ ) or partial BAR domain (GFP-F1, aa 172-352) in Triton X-100-extracted HeLa cells (Figure 6A and 6B). These results indicate that a small fraction of endophilin A1 is resistant to detergent extraction, suggesting that endophilin A1 may associate with the cytoskeleton.

To further characterize possible endophilin A1-cytoskeleton interactions, we examined the effect of latrunculin A, a sponge toxin that binds to globular actin (G-actin) with high affinity and disrupts filamentous actin (F-actin) [30], on the amount of GFP-endophilin A1 in the Triton $\mathrm{X}$-100-insoluble fraction. While there was no significant

Figure 5 Endophilin A1 and p140Cap promote dendritic spine stabilization. (A) Cultured hippocampal neurons were transfected with shRNA constructs coexpressing GFP and shRNA at DIV16-17 followed by live imaging at DIV21. Shown are timelapse images. Right panels are insets of boxed areas in the left panels at different time points. Arrows, protrusions remained throughout the time points; Filled arrowheads, protrusions formed at the time points; Open arrowheads, protrusions eliminated at the time points. (B) Quantitative analysis of turnover ratios (number of cells and number of protrusions analyzed per group are as follows: Ctrl-shRNA: 5 cells, 197 protrusions; EENA1-shRNA: 10 cells, 256 protrusions; p140Cap-shRNA: 6 cells, 198 protrusions). (C, D) Percentage of protrusions formed (number of protrusions formed/preexisting number of protrusions) and eliminated (number of protrusions eliminated/preexisting number of protrusions) over a period of $32 \mathrm{~min}$ in neurons from A. Data represent means \pm SEM. (E) Cultured hippocampal neurons were transfected with Ctrl-shRNA construct or cotransfected with constructs encoding EENA1-shRNA and mCherry or mCherry-tagged RNAi-resistant endophilin A1 (indicated by asterisk), or mCherry-tagged p140Cap at DIV16-17 followed by live imaging at DIV21. Shown are time-lapse images. Red fluorescence indicates the cell body of neurons overexpressing mCherry. Right images are insets of boxed areas in left panels at different time points. Arrows, protrusions remained throughout the time points; Filled arrowheads, protrusions formed at the time points; Open arrowheads, protrusions eliminated at the time points. (F) Quantitative analysis of turnover ratios (number of cells and number of protrusions per group are as follows: Ctrl-shRNA: 5 cells, 157 protrusions; EENA1-shRNA + vector: 9 cells, 201 protrusions; EENA1-shRNA + EENA1*: 6 cells, 205 protrusions; EENA1-shRNA + p140Cap: 7 cells, 231 protrusions). (G, H) Percentage of protrusions formed and eliminated over a period of 32 min in cultured hippocampal neurons from $\mathbf{E}$. Data represent means \pm SEM. Scale bars, $10 \mu \mathrm{m}$. 
A
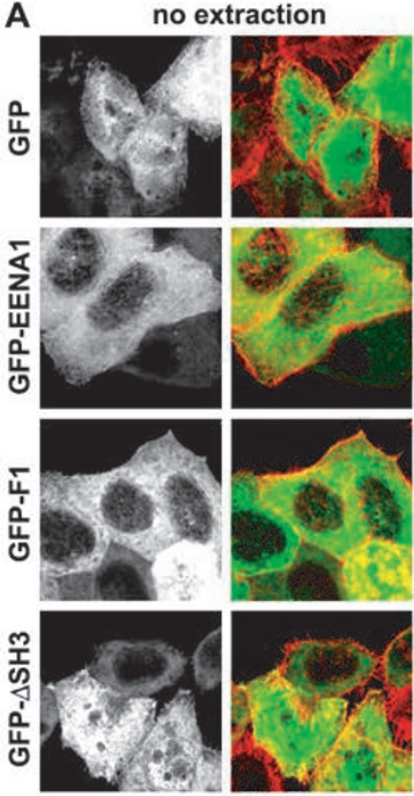

C



Tx-100



D

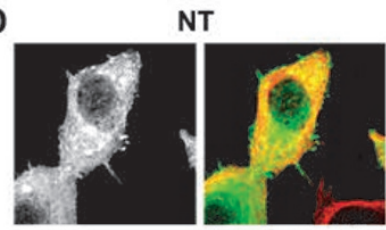

Tx-100

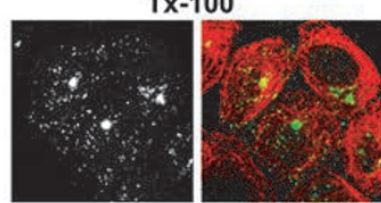


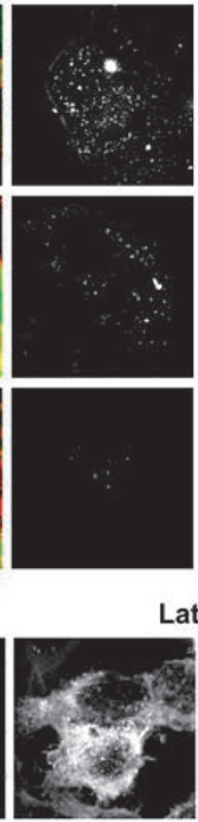

Lat A

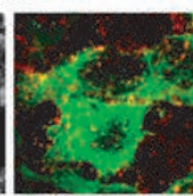

Lat $A+T x-100$

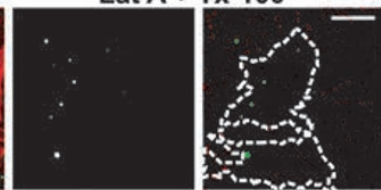

Nocodazole

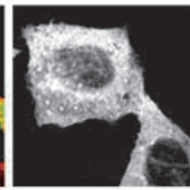

Nocodazole + Tx-100

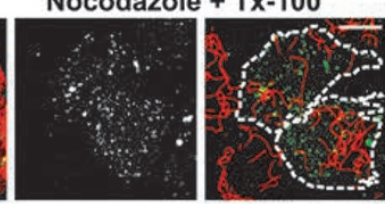

B
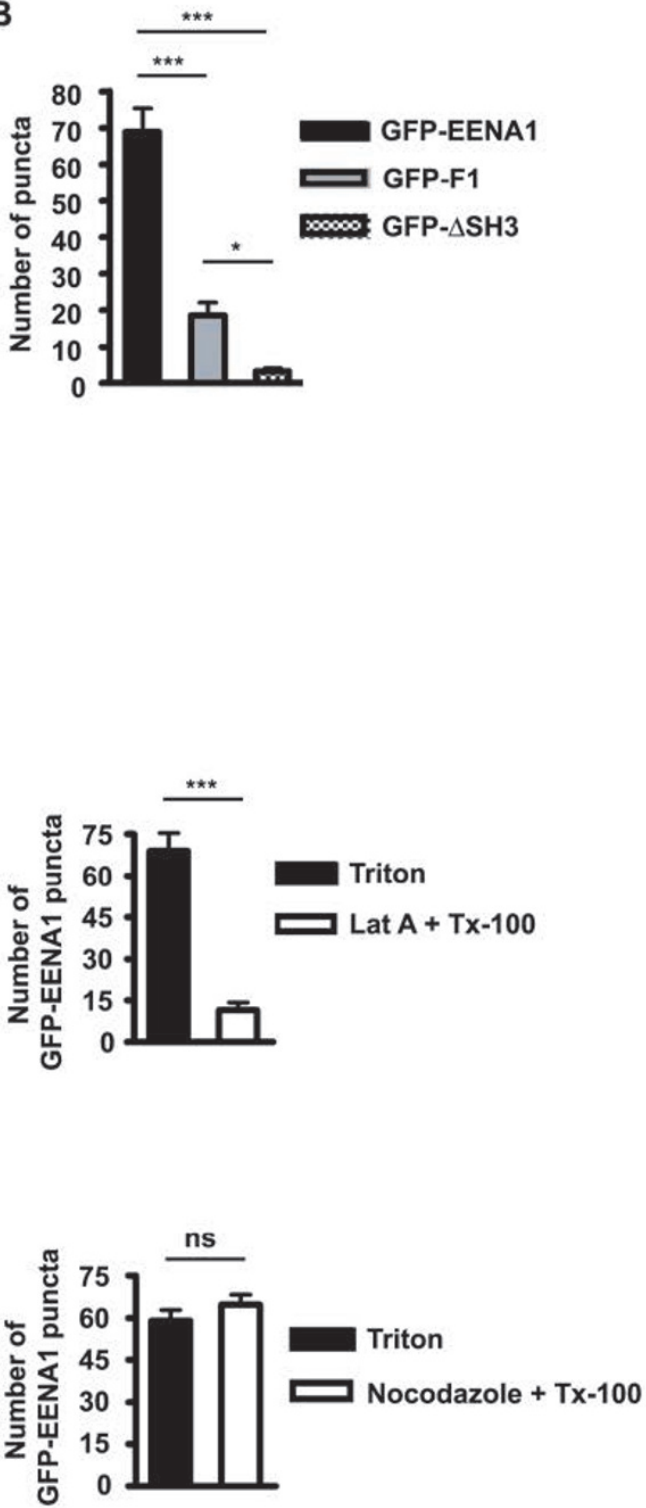

Figure 6 Endophilin A1 associates with F-actin in HeLa cells. (A) HeLa cells transfected with GFP-tagged constructs were extracted with Triton X-100 (Tx-100) 24 h post transfection followed by phalloidin-568 staining for F-actin. (B) Quantification of the number of GFP puncta in HeLa cells in $\mathbf{A},{ }^{* * *} P<0.001,{ }^{*} P<0.05$, one-way ANOVA followed by Newman-Keuls multiple comparison post hoc tests. Number of cells analyzed, GFP-EENA1: 39, GFP-F1: 30, GFP- $\triangle$ SH3: 29. (C) HeLa cells transfected with GFP-tagged endophilin A1 (GFP-EENA1) for $24 \mathrm{~h}$ were pretreated with latrunculin A (Lat A) for 30 min then extracted with Tx-100 followed by phalloidin-568 staining. NT, non-treated. Right panel is the quantification of the number of GFP-EENA1 puncta in HeLa cells, ${ }^{* *} P<0.001$, unpaired $t$-test. Number of cells analyzed, Tx-100: 39, Lat A + Tx-100: 31 . (D) HeLa cells transfected with GFP-EENA1 for $24 \mathrm{~h}$ were pretreated with nocodazole for 30 min then extracted with Tx-100 followed by immunostaining with antibodies against $\alpha$-tubulin. Right panel is the quantification of the number of GFP-EENA1 puncta in HeLa cells. ns, not significant, unpaired $t$-test. Number of cells analyzed, Tx-100: 28, Nocodazole + Tx-100: 31 . Scale bar, $10 \mu \mathrm{m}$. 
A

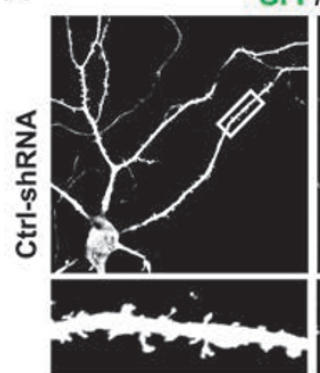

GFP/Lifeact-mCherry/Merge
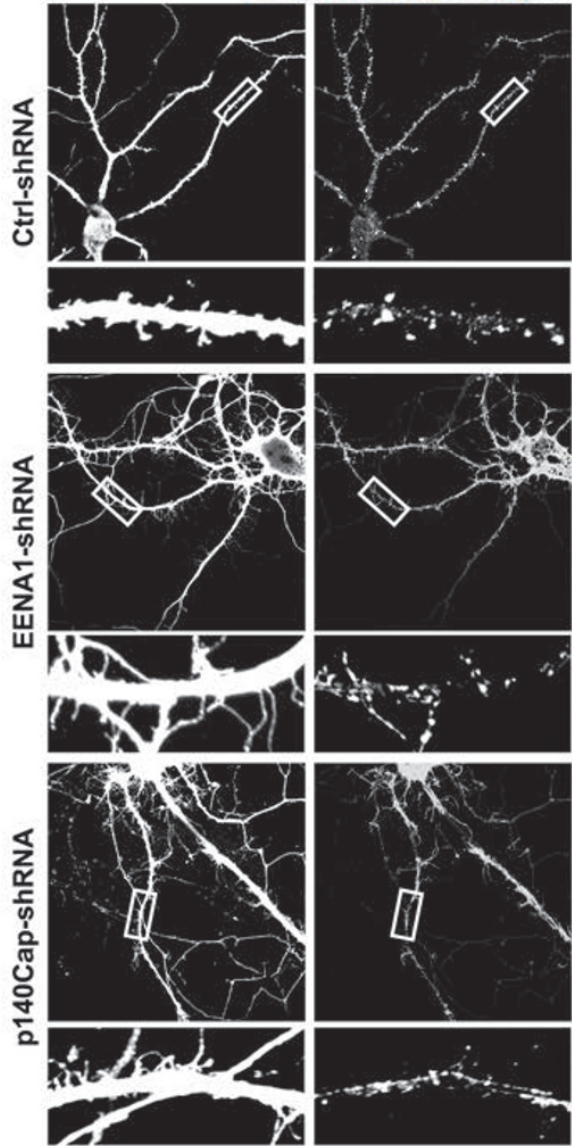

C

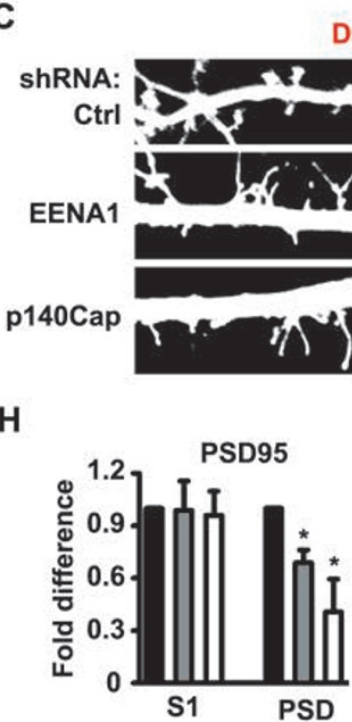

DsRed/p140Cap/Merge
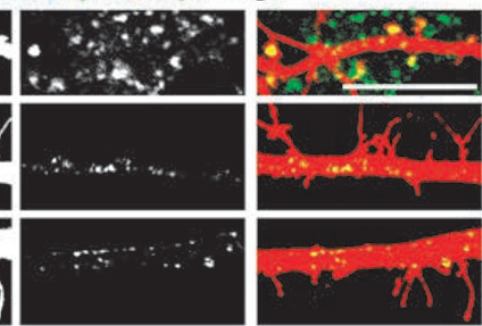

I



B

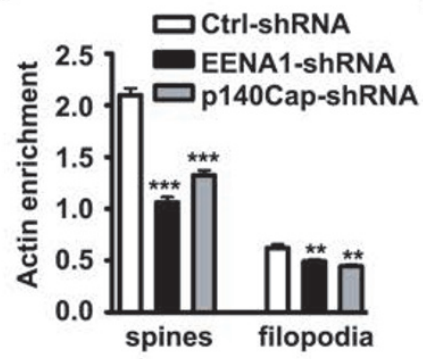

D

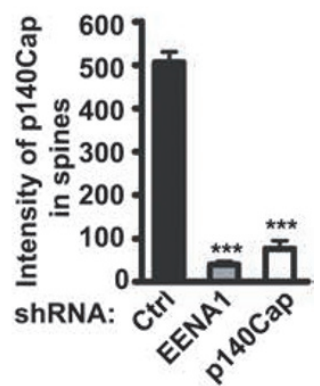

E


$0 \div-\infty-d r e b r i n$

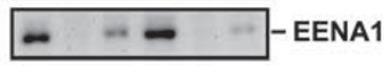

$-0=-$ SYP



$\mathbf{F}$

G
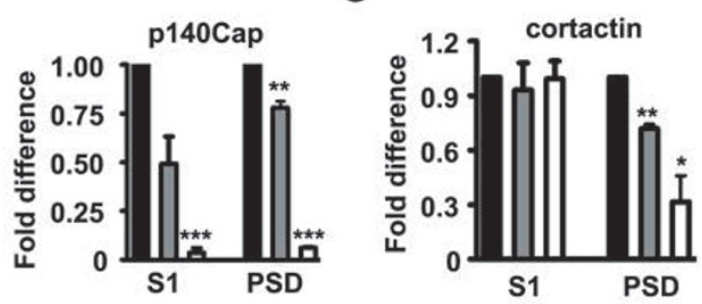

J

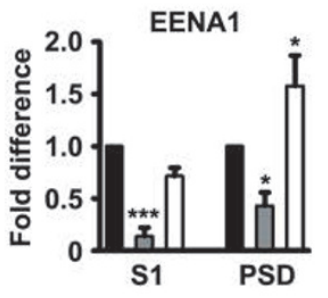

Ctrl-shRNA

EENA1-shRNA

p140Cap-shRNA change in the total level of GFP-endophilin A1, Latrunculin A treatment caused a decrease in the amount of GFP-endophilin A1 and a concomitant decrease in the amount of actin in the Triton X-100-insoluble fraction (Figure 6C). In contrast, nocodazole, a microtubule-de- stabilizing drug [31], had no effect on the amount of GFP-endophilin A1 in the Triton X-100-insoluble fraction (Figure 6D). These data indicate that a small fraction of endophilin A1 is associated with the actin cytoskeleton in cells. 
Figure 7 Endophilin A1 regulates spine morphogenesis via modulating actin cytoskeleton reorganization. (A) Hippocampal neurons were co-transfected with construct coexpressing GFP and shRNA and construct expressing Lifeact-mCherry at DIV16-17 followed by immunostaining with antibodies against GFP and mCherry at DIV21. Shown are representative confocal images. Lower images are insets of boxed areas in upper panels. Scale bar, $10 \mu \mathrm{m}$. (B) Quantification of actin enrichment in dendritic protrusions in A (number of cells and number of protrusions per group are as follows: Ctrl-shRNA: 7 cells, 324 spines, 149 filopodia; EENA1-shRNA: 11 cells, 166 spines, 384 filopodia; p140Cap-shRNA: 10 cells, 253 spines, 323 filopodia.). All values are shown as mean \pm SEM. Statistical test: ${ }^{* *} P<0.001$, ${ }^{* \star} P<0.01$; one-way ANOVA followed by Dunnett's multiple-comparison post hoc tests. (C) Hippocampal neurons were transfected with shRNA constructs coexpressing DsRed and shRNA at DIV16-17 followed by immunostaining with antibodies to DsRed and p140Cap at DIV21. Shown are representative confocal images. Scale bar, $10 \mu \mathrm{m}$. (D) Quantification of p140Cap fluorescence intensity in spines of transfected neurons in $\mathbf{C}$ (number of cells and number of spines per group are as follows: Ctrl-shRNA: 6 cells, 174 spines; EENA1-shRNA: 7 cells, 90 spines; p140Cap-shRNA: 5 cells, 43 spines). All values are shown as mean \pm SEM. Statistical test: ${ }^{* * *} P<0.001$; one-way ANOVA followed by Dunnett's multiple-comparison post hoc tests. (E) Cultured hippocampal neurons were infected with lentivirus expressing shRNA targeting endophilin A1 or p140Cap on DIV9-11, lysed and fractionated on DIV18-20 for immunoblotting analysis. Shown is a representative immunoblot. (F-J) Quantification results of proteins in the homogenates (S1) and in the PSD fraction in E. Statistical test: ${ }^{* * *} P<0.001,{ }^{* *} P<0.01,{ }^{*} P<0.05$; one-way ANOVA followed by Dunnett's multiple-comparison post hoc tests. $n=4$ independent experiments.

Cortactin is an F-actin-binding protein and an activator of the Arp2/3 actin nucleation machinery [32, 33]. Previous studies show that cortactin downregulation results in spine depletion, whereas its overexpression causes spine elongation [34]. It is also reported that overstimulation of NMDA receptors removes cortactin and thereby collapses dendritic spines, whereas stimulation with neurotrophic factors redistributes cortactin from dendritic shafts to spines to solidify synaptic transmission [35]. As p140Cap is able to regulate cortactin phosphorylation by Src and associate with cortactin in neurons [26, 36, 37], next we investigated whether endophilin A1 regulates reorganization of the actin cytoskeleton to drive spine morphogenesis and whether cortactin acts downstream of the endophilin A1-p140Cap pathway.

To test whether silencing of endophilin A1 affects F-actin enrichment in dendritic spines, we transfected neurons with construct overexpressing LifeAct-mCherry, an F-actin-binding protein (Figure 7A). To quantify enrichment of F-actin in protrusions, we measured the fluorescence mean intensity of LifeAct-mCherry within the center of protrusions and normalized each measurement by the fluorescence signal along the adjacent dendritic shaft. Quantitative results showed that there was about 2 -fold decrease in the intensity of LifeAct-mCherry in spines of neurons silenced for endophilin A1 or p140Cap than that in control (Figure 7B), indicating a decrease in F-actin enrichment in spines by downregulation of the endophilin A1-p140Cap pathway. Moreover, immunofluorescence staining showed that knockdown of endophilin A1 also caused a decrease in the intensity of p140Cap signals in spines (Figure 7C and 7D). Consistently, immunoblotting analysis detected a decrease in p140Cap protein levels in the PSD fraction of endophilin A1-silenced neurons (Figure 7E and 7F). Moreover, knock- down of endophilin A1 or p140Cap reduced the protein levels of cortactin and PSD95 but not drebrin, another cytoskeleton regulator, in the PSD fraction (Figure 7E, 7G-7J). Together, these data indicate that endophilin A1 regulates the distribution of both p140Cap and cortactin in dendritic spines to modulate enrichment of F-actin during spine morphogenesis.

To further determine whether the endophilin A1p140Cap pathway regulates spine morphogenesis, synapse formation and function through cortactin, we analyzed spine morphology and synapse formation of cultured hippocampal neurons co-transfected with endophilin A1-shRNA and Flag-tagged cortactin expression constructs. The defects in spine morphology and synapse formation indicated by PSD95 or SYP puncta on dendrites of endophilin A1-silenced neurons were fully rescued by overexpression of cortactin (Figure 8A-8H). Furthermore, the decreased frequency of mEPSCs induced by endophilin A1 knockdown was also rescued by overexpression of p140Cap or cortactin (Figure 8I-8K), indicating that endophilin A1-p140Cap regulates reorganization of actin cytoskeleton through cortactin in spine morphogenesis, synapse formation and synaptic function.

\section{Discussion}

Previous studies have established a role of endophilin A1 in the recycling of synaptic vesicles at the presynaptic site. In this study, we have identified a novel function of endophilin A1 in dendritic spines. We show that reduction of endophlin $\mathrm{A} 1$ expression causes defects in morphogenesis and stability of dendritic spines. We also demonstrate that endophilin A1 regulates spine morphology through p140Cap, an important regulator of actin remodeling, which plays essential roles in dendritic spine 
morphology and synaptic plasticity. Depletion of endophilin A1 not only causes loss of p140Cap and its downstream effector cortactin from the postsynaptic sites, but also loss of $\mathrm{F}$-actin from dendritic protrusions, indicating that endophilin A1 regulates spine morphogenesis and stability through modulation of actin cytoskeleton reorganization.

Previous studies have revealed multiple mechanisms regulating actin dynamics through p140Cap and cortactin in dendritic spines. It was reported that dynamic microtu-
A
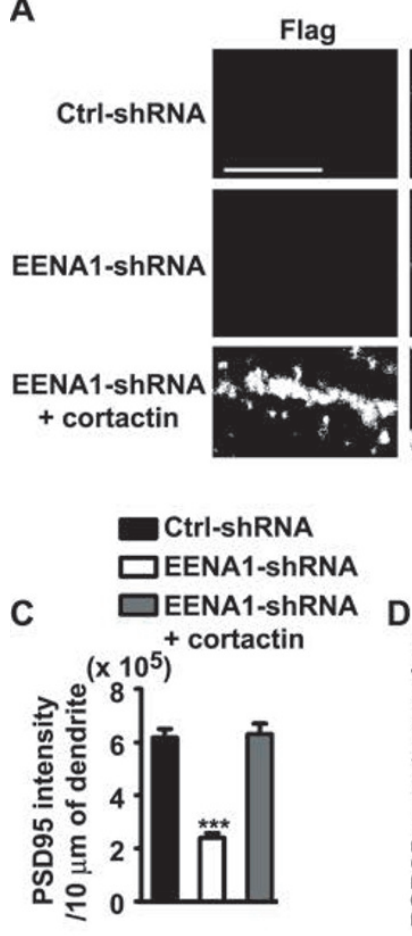

$\mathbf{F}$
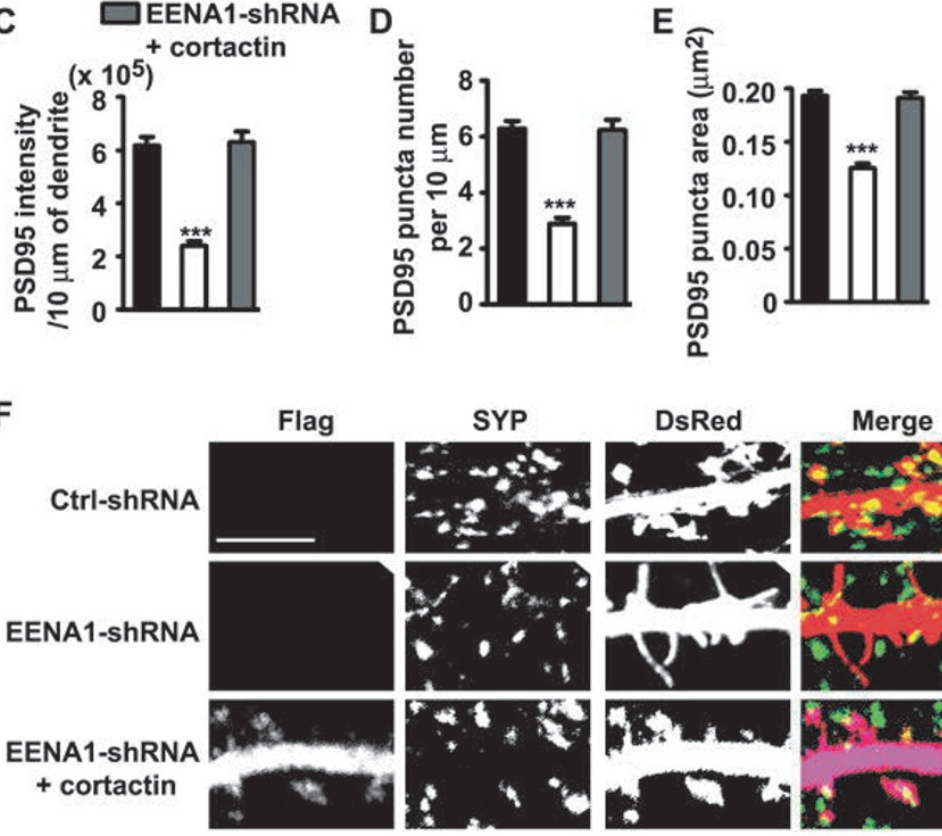

G

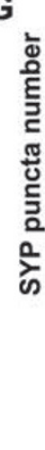

B

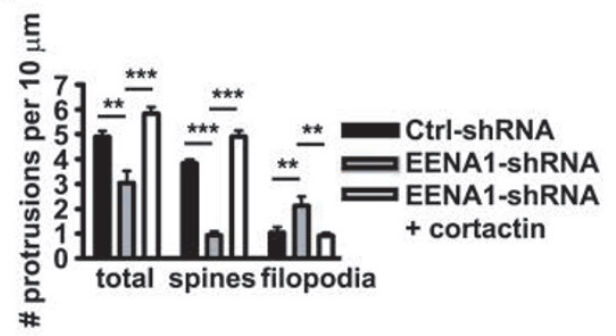

Ctrl-shRNA

$\square E E N A 1-s h R N A$

$\square$ EENA1-shRNA

+ cortactin

H
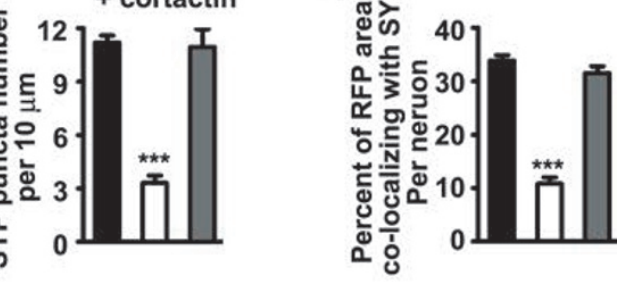

Ctrl-shRNA

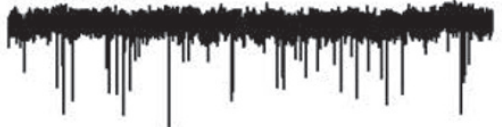

EENA1-ShRNA

Whend

EENA1-shRNA + p140Cap

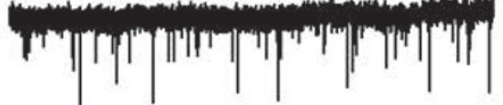

EENA1-shRNA + cortactin



K




bules enter dendritic spines and regulate spine morphology through the microtubule plus-end tracking protein EB3, which regulates the turnover of p140Cap in spines, and that overexpression of the p140Cap-binding protein cortactin could rescue spine shape in EB3-depleted neurons [26]. Partitioning-defective 1b (PAR1b), a serine/ threonine protein kinase believed to regulate microtubule dynamics via phosphorylation of microtubule-associated proteins, was also found to play a role in the maintenance of mature dendritic spine morphology and regulate localization of p140Cap in spines [38]. Most recently, it was reported that SNAP-25, a SNARE protein that is essential for exocytosis of synaptic vesicles at the presynaptic site, also regulates spine formation by recruiting and stabilizing p140Cap in dendritic spines [27]. In this study, we have uncovered yet another mechanism regulating distribution of p140Cap and its downstream effector cortactin in dendrites, which adds another layer of complexity to the regulation of actin cytoskeleton remodeling in dendritic spines. It is conceivable that these mechanisms converge on p140Cap and together they provide precise spatial and temporal controls over the morphogenesis and stabilization of the elaborate structure of dendritic spines to ensure functioning of excitatory synapses in mature neurons. Besides p140Cap, the F-actin-binding protein drebrin has also been shown to bind to EB3 and link dynamic microtubules to F-actin in spines to facilitate microtubule polymerization into the spine $[39,40]$. Intriguingly, depletion of endophilin A1 did not cause changes in drebrin distribution to dendritic spines (Figure 7), indicating that drebrin and p140Cap are regulated through different mechanisms in spines. How these mechanisms are coordinated to regulate the morphology and function of dendritic spines during development remains to be investigated.

Although previous studies have established redundant functions of endophilin A family members in synaptic vesicle recycling at the presynaptic site, our study revealed a novel function of endophilin $\mathrm{A} 1$ at postsynaptic site in the later stages of neuronal development. Interestingly, although endophilin A1 shares more than 70\% homology with endophilin A2 and A3, knockdown of neither endophilin A2 nor A3 affect spine morphogenesis; consistently, overexpression of neither endophilin A2 nor A3 could rescue the spine morphogenesis defects caused by endophilin A1 knockdown, indicating that their functions at the postsynaptic site do not overlap with each other. Since knockout of individual endophilins in mice generated mild phenotypes, and roles of endophilins in vesicle uncoating have been established through analysis of synaptic structures and functions in double and triple knockout mice, functional redundancy and possible compensatory mechanisms for endophilins at the presynaptic site have been proposed [13]. Nevertheless, further investigation of changes in postsynaptic structures and functions in endophilin A1 knockout mice will help dis-

Figure 8 Endophilin A1-p140Cap regulates spine morphogenesis, synapse formation and synaptic function through cortactin. (A) Cultured hippocampal neurons were cotransfected with constructs expressing non-targeting Ctrl-shRNA or EENA1-shRNA and Flag vector, or with constructs expressing EENA1-shRNA and Flag-tagged cortactin at DIV16-17 followed by immunostaining with antibodies against PSD95 (green), Flag (blue) and DsRed at DIV21. Shown are representative confocal images. Scale bar, $5 \mu \mathrm{m}$. (B) Quantitative analysis of dendritic spine protrusion density of transfected neurons in A (number of cells analyzed, Ctrl-shRNA: 15, EENA1-shRNA: 14, EENA1-shRNA + cortactin: 18). More than 500 protrusions were measured for each group. All values are shown as mean \pm SEM. Statistical test: ${ }^{* *} P<0.001,{ }^{* *} P<0.01$; one-way ANOVA followed by Newman-Keuls multiple comparison post hoc tests. (C-E) Quantitative analysis of the fluorescence intensity (C), number (D) and area (E) of PSD95 puncta in dendrites in A (number of cells analyzed, Ctrl-shRNA: 23, EENA1-shRNA: 25, EENA1-shRNA + cortactin: 20; number of puncta analyzed, Ctrl-shRNA: 990, EENA1-shRNA: 578, EENA1-shRNA + cortactin: 923). More than $1200 \mu \mathrm{m}$ of dendrite length were analyzed for each group. All values are shown as mean \pm SEM. Statistical test: ${ }^{* * *} P<0.001$; one-way ANOVA followed by Dunnett's multiple-comparison post hoc tests. (F) Cultured hippocampal neurons were cotransfected with constructs expressing non-targeting Ctrl-shRNA or EENA1-shRNA and Flag vector, or with constructs expressing EENA1-shRNA and Flag-tagged cortactin at DIV16-17 followed by immunostaining with the anti-SYP (green), anti-Flag (blue) and anti-DsRed antibodies at DIV21. Shown are representative confocal images. Scale bar, $5 \mu \mathrm{m}$. (G, H) Quantitative analysis of the SYP puncta number along dendrites $(\mathbf{G})$ or percentage of dendritic area colocalizing with SYP (H) (number of cells analyzed, Ctrl-shRNA: 16, EENA1-shRNA: 18, + cortactin: 19). More than $650 \mu$ m of dendrite length were analyzed for each group. All values are shown as mean \pm SEM. Statistical test: ${ }^{* * *} P<0.001$; one-way ANOVA followed by Dunnett's multiple-comparison post hoc tests. (I) Cultured hippocampal neurons were transfected with Ctrl-shRNA construct or cotransfected with constructs encoding EENA1-shRNA and Flag-tagged p140Cap or Flag-tagged cortactin at DIV9-10 followed by mEPSC recording at DIV14-16. Shown are representative traces of mEPSC recordings. (J) Quantitative analysis of mEPSC frequency and amplitude. $n=3$ independent experiments, 27 neurons. Statistical test: ${ }^{*} P<0.05$; one-way ANOVA followed by Dunnett's multiple-comparison post hoc tests. ns, not significant. (K) Cumulative distribution of mEPSC frequency. Statistical test: $P=0.023$ for EENA1-shRNA versus Ctrl-shRNA, two-sample Kolmogorov-Smirnov test. 
tinguish its role in later stages of neurodevelopment from those of endophilin A2 and A3.

Analysis of mouse brain lysates indicate that protein expression of endophilin A1 increases after birth and stays at high levels throughout neurodevelopment. Given the crucial role of actin dynamics in neuronal morphology not only during spine morphogenesis but also in activity-dependent structural plasticity, and a recently identified function of p140Cap in learning and long-term potentiation/long-term depression using p140Cap-knockout mice [37], our results raise the possibility that endophilin A1 is also involved in regulation of synaptic plasticity and memory.

\section{Materials and Methods}

\section{Ethics statement}

Use of animals was approved by the Animal Care Committee of Institute of Genetics and Developmental Biology, Chinese Academy of Sciences.

\section{Constructs}

The cDNA fragments for mouse endophilin (EEN) A1, A2, $\mathrm{A} 3$ and $\mathrm{B} 1$ were amplified from a mouse brain cDNA obtained by RT-PCR and inserted into pCMV-Tag2B and pCMV-Tag3B. Rat endophilin EENA1 cDNA was amplified from a rat brain cDNA obtained by RT-PCR and inserted into pCMV-Tag3B. EENB1 was inserted into pGEX4T-1 or pET-28a (+). pCMV-Tag2B-EENA1, pCMV-Tag3B-EENA1, pCMV-Tag2B-EENA1 F1 (aa 172-352), pCMV-Tag2B-EENA1 F2 (aa 200-352), pCMV-Tag2B-EENA1 F3 (aa 242-352), pGEX4T-1-EENA1, pGEX4T-1-EENA1 $\triangle$ BAR ( aa 6-242), pGEX4T-1-EENA1 $\Delta \mathrm{SH} 3$ ( $\Delta$ aa 295-346) and pGEX4T1-EENA1 SH3 (aa 295-346) were constructed with mouse endophilin A1 cDNA using standard molecular biology methods. pCMV-Tag2B-EENA1 $\triangle$ BAR ( $\Delta$ aa 6-242), pCMV-Tag2B-EENA1 $\Delta$ SH3 ( aa 295-346), pCMV-Tag2B-EENA1 KKK-EEE (mutation K171, K172 and K173 to E), pCMV-Tag2B-EENA1 BAR$\Delta H 1 I$ ( $\Delta$ aa 1-32 and aa 59-87) and the point mutant constructs of endophilin A1 (299 301308327340 343), were created by site-directed mutagenesis using pCMV-Tag2B-EENA1 as template. EENA1-LentiGFP construct was generated by insertion of mouse EENA1 into the SfiI restriction site of a lentiGFP vector, a generous gift from Dr Xinyu Zhao (University of Wisconsin-Madison School of Medicine and Public Health, Madison, USA [41, 42]). The shRNA-resistant endophilin A1 expression construct was generated by modifying $5^{\prime}$-GGACTCATTGGACATGGAA-3' (nt 384-402 of mouse endophilin A1 coding region) into 5'-GGACTCTCTTGACATGGAA-3' by site-directed mutagenesis. The shRNA-resistant endophilin A1, F1 and $\triangle \mathrm{SH} 3$ were amplified by PCR and inserted into mCherryC2 or pEGFPC2 vector. The p140Cap cDNA construct was a generous gift from Dr Casper C Hoogenraad (Utrecht University, Utrecht, the Netherlands). Fulllength p140Cap, p140Cap aa 1-1 051, p140Cap aa 351-1 051, PRD1 (aa 351-677), PRD2 (aa 841-1 059) and CT (aa $1052-$ 1217 ) were amplified and cloned into pCMV-Tag2B. pCMVTag2B-p140Cap $\triangle$ PRD1 ( $\Delta$ aa 351-677), pCMV-Tag2B-p140Cap $\Delta$ PRD2 $(\Delta$ aa $869-1051)$, or $\Delta$ PRD1 $+\Delta$ PRD2 $(\Delta$ aa $351-677$ and $\Delta$ aa $869-1051$ ) were generated by site-directed mutagenesis. Full-length p140Cap was subcloned into pCMV-Tag3B or cloned into mCherryC2 vector. p140Cap aa 841-1 059 was amplified and cloned into pET-28a(+). Full-length mouse cortactin cDNA was amplified from a mouse brain cDNA and inserted into $\mathrm{pC}$ MV-Tag2B. Mouse drebrin aa 1-278 was amplified and cloned into pET-28a (+). The LifeAct-mCherry construct was a generous gift from Dr Evelyne Coudrier (Institut Curie, France).

To prepare shRNA constructs, each shRNA contained a $19 \mathrm{nt}$ target sequence, the sense and antisense strands were annealed, and then the annealing oligos were inserted into pLL3.7.4 between XhoI and HpaI sites. All constructs were verified by sequencing. Target sequences for rat endophilin A1 \#1: 5'-GGACTCTTTGGACATGGAA-3'; rat endophilin A1 \#2: 5'-GAAGCGACAAGGAAAGATT-3'; for rat endophilin A2 shRNA: 5'-GAACCTGTGTGACAAGGAT-3'; for rat endophilin A3: 5'-GAGCTAAGCTAGGAATGCT-3'; for rat endophilin B1: 5'-GGTGCCAATTACCTACTTA-3'; for rat p140Cap shRNA \#1: 5'-GACGTGTACACATCACGCA-3'; for rat p140Cap shRNA \#2: 5'-GTCCTGAGTTGGAAGAGAA-3'; for control shRNA, which has no homology to known gene sequences: 5'-GAATGCTCTTACGATGATA-3'.

\section{Antibodies}

Anti-endophilin B1, anti-p140Cap and anti-drebrin antisera were obtained by immunization of rabbit with histidine (His)tagged full-length endophilin B1, p140Cap (aa 841-1 059) and drebrin (aa 1-278) recombinant protein expressed and purified from Escherichia coli, respectively. Antibodies were affinity purified with the antigen immobilized on Amino Link agarose gel beads (Affi-Gel; Bio-Rad Laboratories, Hercules, CA). The following antibodies were obtained from commercial sources: goat anti-endophilin A1 (S-20), endophilin A2 (E-15), endophilin A3 (K17), mouse anti-SYP (D-4) and mouse anti-cortactin (E-4) (Santa Cruz Biotechnology, Santa Cruz, CA); rabbit anti-endophilin A1 (Synaptic Systems GmbH, Germany); rabbit anti-Myc, rabbit and mouse anti-GFP, rabbit and mouse anti-RFP which recognizes DsRed and mCherry (Medical \& Biological Laboratories, Naka-ku Nagoya, Japan); mouse anti-PSD95 (75-028) for immunofluorescence staining (NeuroMab, Davis, CA); monoclonal anti-PSD95 for western blotting (BD Biosciences, San Diego, CA), mouse anti-FLAG M2, mouse anti- $\alpha$-tubulin and mouse anti- $\beta$-actin (Sigma-Aldrich, St. Louis, MO); secondary antibodies for immunofluorescence staining were from Molecular Probes (Invitrogen, Carlsbad, CA). Colloidal gold secondary antibodies for immunoEM were from Jackson ImmunoResearch Laboratories (West Grove, PA).

\section{Cell culture, transfection and viral infection}

HEK293T and HeLa cells were cultured in DMEM supplemented with $10 \%$ fetal bovine serum (FBS). Transfections were performed using VigoFect according to the manufacturer's instructions (Vigorous Co., Bangkok, Thailand). Cells were harvested 24-48 h post transfection. For Triton X-100 extraction, HeLa cells cultured on coverslips with or without latrunculin A $(0.8 \mu \mathrm{M})$ or nocodazole $(10 \mu \mathrm{M})$ pretreatment for $30 \mathrm{~min}$ were washed twice with PBS and incubated in the buffer (4 M glycerol, $25 \mathrm{mM}$ PIPES, $1 \mathrm{mM}$ EGTA, $1 \mathrm{mM} \mathrm{MgCl}_{2}$, and $0.5 \%$ Triton X-100, $\mathrm{pH}$ 6.9) at room temperature (RT) for $5 \mathrm{~min}$ [43], followed by fixation and 
immunostaining with phalloidin or antibodies against tubulin.

Primary neuronal cultures from rat hippocampi or cortex were prepared as described previously [44]. Briefly, hippocampi and cortex were dissected from embryonic day 17.5 (E17.5) Sprague Dawley (SD) rat, dissociated with $0.125 \%$ trypsin in Hank's balanced salt solution without $\mathrm{Ca}^{2+}$ and $\mathrm{Mg}^{2+}$ at $37^{\circ} \mathrm{C}$ for $20 \mathrm{~min}$, triturated in DMEM, 10\% F12, and 10\% FBS. Hippocampal neurons were plated on poly-D-lysine-treated coverslips in 24-well plates at a density of $3 \times 10^{4}$ cells/well. Cortical neurons were plated in poly-D-lysine-treated six-well plates at a density of $1.5 \times 10^{6}$ cells/ well. The medium was changed to the serum-free Neurobasal (NB) medium supplemented with 2\% B27 supplement and GlutaMAX (Gibco, Invitrogen) $4 \mathrm{~h}$ after plating.

For neuronal morphology and immunofluorescence staining, neuronal transfections were performed using Lipofectamine 2000 according to the manufacturer's instructions (Invitrogen) on 16-17 DIV after plating. Briefly, DNA $(1.2 \mu \mathrm{g} /$ well) was mixed with $1 \mu \mathrm{l}$ Lipofectamine 2000 in $250 \mu \mathrm{l} \mathrm{NB}$ medium, incubated for $20 \mathrm{~min}$ and then added to the neurons in $\mathrm{NB}$ at $37{ }^{\circ} \mathrm{C}$ in $5 \% \mathrm{CO}_{2}$ for $1 \mathrm{~h}$. Neurons were then rinsed with $\mathrm{NB}$ and incubated in the original medium at $37{ }^{\circ} \mathrm{C}$ in $5 \% \mathrm{CO}_{2}$ for $4-5$ days. For co-transfection, neurons were transfected with $1.8 \mu \mathrm{g}$ of plasmid DNA consisting of 0.8 $\mu \mathrm{g}$ shRNA plasmid and $1.0 \mu \mathrm{g}$ of a rescue plasmid or consisting of $0.9 \mu \mathrm{g}$ shRNA plasmid and $0.9 \mu \mathrm{g}$ LifeAct-mCherry.

For western blotting, neurons were infected with Lentivirus prepared from HEK293T cells for 4-6 h at DIV 9-11, Neurons were then washed with NB and transferred to the original medium at $37^{\circ} \mathrm{C}$ in $5 \% \mathrm{CO}_{2}$ and harvested after 7-9 days.

\section{Preparation of recombinant lentivirus}

Lentivirus production was as described previously [42]. Briefly, lentiviral transfer vector, packaging plasmid DNA and envelope plasmid DNA were co-transfected into cultured 293T cells using VigoFect. The medium containing lentivirus was collected $48 \mathrm{~h}$ post transfection, pooled, filtered through a $0.22-\mu \mathrm{m}$ filter, and concentrated at $33000 \mathrm{rpm}$ for $90 \mathrm{~min}$ at $4{ }^{\circ} \mathrm{C}$ using a MLA-55 Rotor (Beckman). The virus was resuspended in $400 \mu \mathrm{l}$ of NB media and incubated overnight at $4{ }^{\circ} \mathrm{C}$ and aliquots were stored at $-80{ }^{\circ} \mathrm{C}$.

Immunofluorescence staining, image acquisition and analysis

Hippocampal neurons were fixed in 4\% paraformaldehyde $/ 4 \%$ sucrose in phosphate-buffered saline (PBS) at RT for $15 \mathrm{~min}$, and then permeabilized in $0.4 \%$ Triton X-100 in PBS for 10 min. After blocking with $1 \%$ BSA in PBS containing $0.4 \%$ Triton $\mathrm{X}-100$ for $1 \mathrm{~h}$ at RT, neurons were incubated with primary antibodies for $1 \mathrm{~h}$ at RT or overnight at $4{ }^{\circ} \mathrm{C}$, and appropriate secondary antibodies conjugated with Alexa Fluor 488, Alexa Fluor 555, or Alexa Fluor 647 were used for detection. Confocal images were collected using the Spectral Imaging Confocal Microscope Digital Eclipse C1Si (Nikon, Tokyo, Japan) with a $100 \times$ Plan Apochromat VC NA 1.40 oil objective. Images were $\mathrm{z}$ projections of images taken at 0.15-0.2 $\mu \mathrm{m}$ step intervals. The number of planes, typically 5-7, was chosen to cover the entire dendrite from top to bottom. For structured illumination microscopy, secondary antibodies conjugated with Alexa Fluor 568 were used and images were captured as described by Niu et al. [45] on the Delta Vision OMX V4 imaging system (Applied Precision) with a $60 \times 1.4$ oil objective (Olympus UPlanSApo). Three dimensional images were reconstructed from $\mathrm{z}$ stacks using the Surpass Volume mode of the Imaris software (Bitplane AG).

For the morphometric analysis of dendritic protrusions, we used GFP or DsRed as an unbiased cell-fill. Maximum image projections and three-dimensional projections used in spine density calculations were rendered with the NIS-Elements AR software (Nikon) from confocal z-series images. Dendrites of 40-120 $\mu \mathrm{m}$ region from cell body were selected per neuron for analysis. Spine and filopodium density were determined by manually counting and classifying protrusions as "spine" and "filopodium" along a minimum of $70 \mu \mathrm{m}$ of dendrite per cell and was expressed per 10 $\mu \mathrm{m}$ length of dendrite. Spine length and width were measured as described previously using the measurement tool in NIS-Elements AR software [46] and protrusions were classified based on the ratio of spine head width to protrusion length according to the following ratios: the spine whose width was equal to or more than half the size of its length was defined as standard "spine". The protrusion whose width was smaller than half the size of its length was defined as "filopodia" [26]. For statistical analyses, the lamellipodia-like structures (defined by a protrusion width of $>2.5 \mu \mathrm{m}$ ) or protrusions length $>5 \mu \mathrm{m}$ were excluded [47]. All morphological experiments were repeated at least three times with an $n>12$ for individual experiments.

To quantify PSD95 puncta, the colocalization of SYP with dendrites, the colocalization of endophilin A1 with PSD95 or p140Cap, and the intensity of p140Cap in spines, the DsRed fluorescence was used to label neurons transfected with control, EENA1 or p140Cap shRNA construct. The DsRed-labeled dendrites or spines were outlined manually. The intensity or colocalization was analyzed with the NIS-Elements AR software. Puncta were defined by thresholding images at two times background (staining within the dendritic shaft). Colocalization was calculated with Mander's overlap coefficient. Values for colocalization analysis represent mean \pm SEM.

Protrusion motility of dissociated hippocampal neurons was imaged by confocal microscopy using a $100 \times$ oil-immersion objective in Nunc Lab-Tek II Chamber Slide (Thermo Fisher Scientific Inc. Waltham, MA, USA). To reduce photobleaching, laser levels were set as low as possible to still permit detection of protrusions, the pinhole was opened to $\mathrm{M}$, and scanning was minimized by taking $0.2-0.3 \mu \mathrm{m}$ step to create $\mathrm{z}$ projections. Images were collected every 2 min for 30-32 min for spine dynamics experiments. For each session, the time between images was chosen to minimize laser exposure of the field while allowing resolution of protrusions. Images were analyzed in z-series images for the formation or elimination of each spine. TOR, the fraction of spines appearing and disappearing between successive imaging sessions, were calculated as TOR $=\left(\mathrm{N}_{\text {gained }}+\mathrm{N}_{\text {lost }}\right) /\left(2 \times \mathrm{N}_{\text {total }}\right)$. For all analyses, statistical measures were collected from a minimum of three independent experiments.

\section{Western blot and immunoprecipitation}

For developmental expression analysis, cerebral cortices were dissected from E14, E16, E18, P0, P7, P21, and 2-month-old mice and rinsed once in ice-cold PBS, $\mathrm{pH}$ 7.4. Frozen samples were homogenized in lysis buffer (50 mM Tris-Cl, $\mathrm{pH} 7.4,150 \mathrm{mM} \mathrm{NaCl}$, $5 \mathrm{mM}$ EDTA, $0.5 \%$ Triton X-100) supplemented with protease inhibitors. $20 \mu \mathrm{g}$ of protein was loaded in each lane for subsequent western blot analysis. 
For immunoprecipitation, DIV18-21 neurons were washed with ice-cold PBS and lysed with lysis buffer A $(0.05 \%$ (vol $/ \mathrm{vol}) \mathrm{NP}$ 40, $15 \mathrm{mM}$ Tris- $\mathrm{HCl}, \mathrm{pH} 7.4,50 \mathrm{mM} \mathrm{NaCl}$ ) supplemented with protease inhibitors for endogenous IP, or HEK293 were lysed with lysis buffer B (0.1\% (vol/vol) NP-40, 50 mM Tris-HCl, $\mathrm{pH} 7.4$, $150 \mathrm{mM} \mathrm{NaCl}, 5 \mathrm{mM}$ EDTA) supplemented with protease inhibitors. Lysates were then centrifuged at $12000 \times \mathrm{g}$ for $15 \mathrm{~min}$ at $4{ }^{\circ} \mathrm{C}$. For Flag IP, the supernatants were incubated with anti-Flag Affinity Gel (Sigma-Aldrich) at $4{ }^{\circ} \mathrm{C}$ for $2-4 \mathrm{~h}$. For other IPs, antibodies $(1 \mu \mathrm{g})$ were added to cell lysates and incubated at $4{ }^{\circ} \mathrm{C}$ for $2 \mathrm{~h}$, followed by incubation with Protein A/G PLUS-agarose (Santa Cruz) pre-equilibrated in lysis buffer overnight at $4{ }^{\circ} \mathrm{C}$. Precipitates were washed five times with lysis buffer. Immunoprecipitates were eluted from the agarose by boiling in $2 \times$ SDS Gel loading buffer (100 mMTris-Cl, pH 6.8, 4\% SDS, 0.2\% romophenol blue, $20 \%$ (vol $/ \mathrm{vol}$ ) glycerol, $10 \%$ (vol/vol) 2-mercaptoethanol) and subjected to SDS-PAGE and immunoblotting. Immunoblots were imaged with an Epichemi3 Darkroom system (UVP BioImaging Systems, Upland, CA). For densitometric analysis, immunoreactive bands were quantified using ImageJ (National Institutes of Health, Bethesda, MD). All experiments were carried out independently at least three times.

\section{PSD preparations}

Cytosol, synaptosome, synaptosomal membrane and PSD fractions from mouse brain or cultured cortical neurons were prepared using the previously described procedure with slight modifications $[26,48,49]$. In brief, cortex and hippocampi were homogenized on ice using 20 strokes of a Teflon-glass homogenizer in $1 \mathrm{ml}$ of HEPES-buffered sucrose (0.32 M sucrose, $4 \mathrm{mM}$ HEPES, $\mathrm{pH} 7.4)$ containing freshly added protease inhibitors, or $10^{7}$ cortical neurons in $10 \mathrm{~cm}$ dishes were grown for 21 days and cells were scraped into $900 \mu 1$ HEPES-buffered sucrose, homogenized with a syringe (20-30 strokes), then centrifuged at 800-1 $000 \times g$ at $4{ }^{\circ} \mathrm{C}$ to remove the pelleted nuclear fraction (P1). Supernatant (S1) was centrifuged at $10000 \times g$ for 15 min to yield the crude synaptosomal pellet (P2) and pellet was washed once in $1 \mathrm{ml}$ HEPES-buffered sucrose. P2 was lysed by hypoosmotic shock in $900 \mu \mathrm{l}$ ice-cold $4 \mathrm{mM}$ HEPES, $\mathrm{pH} 7.4$ plus protease inhibitors, homogenized by pipetting and mixed for $30 \mathrm{~min}$ at $4{ }^{\circ} \mathrm{C}$. The lysate was centrifuged at $25000 \times g$ for 20 min to yield supernatant (S3, crude synaptic vesicle fraction) and pellet (P3, lysed synaptosomal membrane fraction). To prepare the PSD fraction, P3 was resuspended in $900 \mu \mathrm{l}$ of ice-cold $50 \mathrm{mM}$ HEPES, pH 7.4, $2 \mathrm{mM}$ EDTA, plus protease inhibitors and $0.5 \%$ Triton X-100, rotated for $15 \mathrm{~min}$ at $4{ }^{\circ} \mathrm{C}$ and centrifuged at $32000 \times \mathrm{g}$ for $20 \mathrm{~min}$ to obtain the PSD pellet. PSD pellets were resuspended in $50 \mu 1$ ice-cold 50 mM HEPES, pH7.4, 2 mM EDTA plus protease inhibitors.

\section{Electrophysiology}

Whole-cell patch-clamp recordings of miniature EPSCs were obtained from transfected cultured hippocampal neurons on DIV14-16 at the density of $5.0 \times 10^{4} /$ well. During recordings, cells were bathed in an external solution containing (in $\mathrm{mM}$ ): 150 $\mathrm{NaCl}, 4 \mathrm{KCl}, 2 \mathrm{CaCl}_{2}, 1 \mathrm{MgCl}_{2}, 10$ glucose, and 10 HEPES pH 7.4, $1 \mathrm{mM}$ tetrodotoxin, $100 \mu \mathrm{M}$ picrotoxin. Recording pipettes were filled with the intracellular solution containing (in $\mathrm{mM}$ ): 145 $\mathrm{KCl}, 5 \mathrm{NaCl}, 5$ EGTA, 10 HEPES, $1 \mathrm{MgCl}_{2}, 4 \mathrm{MgATP}$ and 0.3 $\mathrm{Na}_{2}$ GTP. Recordings were performed at RT in voltage clamp mode at holding potential of $-60 \mathrm{mV}$ using a Multiclamp 700B amplifier (Molecular Devices) and Clampex 10.2.0.12 software (Axon Instruments, Foster City, CA, USA). Series resistance below $20 \mathrm{MO}$ was monitored for consistency during recordings. Cells in culture with leak currents $250 \mathrm{pA}$ were excluded from analysis. Signals were amplified, sampled at $10 \mathrm{kHz}$, filtered to $3 \mathrm{KHz}$, and analyzed using Clampfit (Axon Instruments, Foster City, CA, USA).

\section{GST pull-down assays}

GST-fused EENA1, EENA1 $\Delta$ BAR, EENA1 $\Delta$ SH3, EENA1-SH3 were expressed and purified from E. coli. p140Cap constructs were expressed in HEK293 cells for $48 \mathrm{~h}$ and lysates were prepared in a buffer $(50 \mathrm{mM}$ Tris- $\mathrm{HCl}, \mathrm{pH} 7.5,150 \mathrm{mM}$ $\mathrm{NaCl}, 1 \%$ Triton $\mathrm{X}-100,1 \mathrm{mM}$ DTT) plus protease inhibitors. Cell lysates were centrifuged at $12000 \times \mathrm{g}$ for $15 \mathrm{~min}$ at $4{ }^{\circ} \mathrm{C}$ and the supernatant was incubated with individual GST-fused proteins for $2 \mathrm{~h}$ at $4{ }^{\circ} \mathrm{C}$. Beads were washed five times with a buffer containing $20 \mathrm{mM}$ Tris- $\mathrm{HCl}, \mathrm{pH} 7.5,150 \mathrm{mM} \mathrm{NaCl}, 0.5 \%$ Triton $\mathrm{X}-100$ and boiled in SDS sample buffer. Bound proteins were analyzed by immunoblotting.

\section{Immuno-electron microscopy}

Tissue preparation and immuno-electron microscopy were conducted as described [50]. Briefly, adult mouse was anesthetized with $2 \%$ pentobarbital sodium then perfusion-fixed with cold solution containing 0.4\% glutaraldehyde (Cat\#16220; Electron Microscopy Sciences, Hatfield, PA), 4\% PFA (Cat\#157-8, Electron Microscopy Sciences) in phosphate buffer (PB; $0.1 \mathrm{M}, \mathrm{pH}$ 7.4). The brain was seperated immediately and immersed in the same fixative for $4 \mathrm{~h}$ at $4{ }^{\circ} \mathrm{C}$ and then stored in $0.1 \mathrm{M}$ PB buffer. Hippocampus blocks were collected in $0.1 \mathrm{M}$ PB and postfixed for $30 \mathrm{~min}$ with $1 \%$ osmium tetroxide $\left(\mathrm{OsO}_{4}\right)$ and rinsed with $\mathrm{PB}$, the blocks were dehydrated with gradient ethanol, infiltrated and embedded with LR White (Cat\#14381-UC, Electron Microscopy Sciences), polymerized at $37{ }^{\circ} \mathrm{C}$ for $12 \mathrm{~h}, 42{ }^{\circ} \mathrm{C}$ for $12 \mathrm{~h}$ and $50{ }^{\circ} \mathrm{C}$ for $24 \mathrm{~h}$. Ultrathin sections $(70 \mathrm{~nm})$ were collected on 200 -mesh nickel grids coated with Formvar membrane, rinsed and blocked with BSA/PB (1\% BSA in $0.1 \mathrm{M} \mathrm{PB})$ and incubated with anti-endophilin A1 antibody (1:5) and/or anti-p140Cap antibody (1:10) in BSA/PB overnight at $4{ }^{\circ} \mathrm{C}$, washed and incubated with $12 \mathrm{~nm}$ gold Donkey anti-Goat IgG (1:20, Jackson) and/or $18 \mathrm{~nm}$ gold Donkey anti Rabbit-IgG (1:15, Jackson). Sections were examined under a JEM-1400 transmission electron microscope (JEOL, Tokyo, Japan) and images were captured with a Gatan CCD $(4 \mathrm{k} \times 3.7 \mathrm{k}$ pixles, USA). An ultrastructure was considered as positive for the protein studied when a minimum of two gold particles (one size or both sizes) was found.

\section{Statistical analysis}

All data are presented as the mean \pm SEM. GraphPad Prism 5 (GraphPad Software, La Jolla, CA) was used for statistical analysis. The mEPSC data were plotted using IGOR Pro (Wavemetrics, Inc). For two-sample comparisons vs controls, unpaired Student's $t$-test was used except where noted. Kolmogorov-Smirnoff (K-S) test was used for cumulative distributions. One-way analysis of variance with a Dunnett's multiple-comparison or Newman-Keuls multiple comparison hoc test was used to evaluate statistical significance of three or more groups of samples. $P$ value $<0.05$ was considered statistically significant. 


\section{Acknowledgments}

We are indebted to our colleagues for providing reagents (Dr Xinyu Zhao, University of Wisconsin, Madison, USA; Dr Casper C Hoogenraad, Utrecht University, The Netherlands and Dr Evelyne Coudrier, Institut Curie, France) and for critical comments on the manuscript (Dr Tieshan Tang, Institute of Zoology, Chinese Academy of Sciences, China). We are grateful to Dr Lan Bao (Institute of Biochemistry and Cell Biology, Chinese Academy of Sciences, China), Dr Hongli Feng, Dr Ping Zhu and Lei Sun (Institute of Biophysics, Chinese Academy of Sciences, China) for technical assistance and advices on electron microscopy. This work was supported by the National Basic Research Program (2014CB942802 to J-JL and CZ, 2011CB965002 to J-JL), the National Natural Science Foundation of China (31325017, 30830059 to J-JL, 31222025, 31171025 to CZ) and CAS Key Project (KSCX2-EW-R-05 to J-JL). YY was supported by Young Scientist Award from the National Natural Science Foundation of China (30900718).

\section{References}

1 Kasai H, Matsuzaki M, Noguchi J, Yasumatsu N, Nakahara H. Structure-stability-function relationships of dendritic spines. Trends Neurosci 2003; 26:360-368.

2 Dailey ME, Smith SJ. The dynamics of dendritic structure in developing hippocampal slices. J Neurosci 1996; 16:29832994.

3 Ziv NE, Smith SJ. Evidence for a role of dendritic filopodia in synaptogenesis and spine formation. Neuron 1996; 17:91-102.

4 Calabrese B, Wilson MS, Halpain S. Development and regulation of dendritic spine synapses. Physiology (Bethesda) 2006; 21:38-47.

5 Penzes P, Cahill ME, Jones KA, VanLeeuwen JE, Woolfrey KM. Dendritic spine pathology in neuropsychiatric disorders. Nat Neurosci 2011; 14:285-293.

6 Tada T, Sheng M. Molecular mechanisms of dendritic spine morphogenesis. Curr Opin Neurobiol 2006; 16:95-101.

7 Bosch M, Hayashi Y. Structural plasticity of dendritic spines. Curr Opin Neurobiol 2012; 22:383-388.

8 Hotulainen P, Llano O, Smirnov S, et al. Defining mechanisms of actin polymerization and depolymerization during dendritic spine morphogenesis. J Cell Biol 2009; 185:323339.

9 de Heuvel E, Bell AW, Ramjaun AR, Wong K, Sossin WS, McPherson PS. Identification of the major synaptojanin-binding proteins in brain. J Biol Chem 1997; 272:8710-8716.

10 Ringstad N, Nemoto Y, De Camilli P. The SH3p4/Sh3p8/ SH3p13 protein family: binding partners for synaptojanin and dynamin via a Grb2-like Src homology 3 domain. Proc Natl Acad Sci USA 1997; 94:8569-8574.

11 Chowdhury S, Shepherd JD, Okuno H, et al. Arc/Arg3.1 interacts with the endocytic machinery to regulate AMPA receptor trafficking. Neuron 2006; 52:445-459.

12 Rial Verde EM, Lee-Osbourne J, Worley PF, Malinow R, Cline HT. Increased expression of the immediate-early gene arc/arg3.1 reduces AMPA receptor-mediated synaptic transmission. Neuron 2006; 52:461-474.

13 Milosevic I, Giovedi S, Lou X, et al. Recruitment of endophi- lin to clathrin-coated pit necks is required for efficient vesicle uncoating after fission. Neuron 2011; 72:587-601.

14 Llobet A, Gallop JL, Burden JJ, et al. Endophilin drives the fast mode of vesicle retrieval in a ribbon synapse. $J$ Neurosci 2011; 31:8512-8519.

15 Verstreken P, Kjaerulff O, Lloyd TE, et al. Endophilin mutations block clathrin-mediated endocytosis but not neurotransmitter release. Cell 2002; 109:101-112.

16 Schuske KR, Richmond JE, Matthies DS, et al. Endophilin is required for synaptic vesicle endocytosis by localizing synaptojanin. Neuron 2003; 40:749-762.

17 Guichet A, Wucherpfennig T, Dudu V, et al. Essential role of endophilin A in synaptic vesicle budding at the Drosophila neuromuscular junction. EMBO J 2002; 21:1661-1672.

18 Nakano-Kobayashi A, Kasri NN, Newey SE, Van Aelst L. The Rho-linked mental retardation protein OPHN1 controls synaptic vesicle endocytosis via endophilin A1. Curr Biol 2009; 19:1133-1139.

19 Bai J, Hu Z, Dittman JS, Pym EC, Kaplan JM. Endophilin functions as a membrane-bending molecule and is delivered to endocytic zones by exocytosis. Cell 2010; 143:430-441.

20 Verstreken P, Koh TW, Schulze KL, et al. Synaptojanin is recruited by endophilin to promote synaptic vesicle uncoating. Neuron 2003; 40:733-748.

21 Ringstad N, Gad H, Low P, et al. Endophilin/SH3p4 is required for the transition from early to late stages in clathrin-mediated synaptic vesicle endocytosis. Neuron 1999; 24:143-154.

22 Fu X, Yang Y, Xu C, et al. Retrolinkin cooperates with endophilin A1 to mediate BDNF-TrkB early endocytic trafficking and signaling from early endosomes. Mol Biol Cell 2011; 22:3684-3698.

23 Chin LS, Nugent RD, Raynor MC, Vavalle JP, Li L. SNIP, a novel SNAP-25-interacting protein implicated in regulated exocytosis. J Biol Chem 2000; 275:1191-1200.

24 Di Stefano P, Cabodi S, Boeri Erba E, et al. P130Cas-associated protein (p140Cap) as a new tyrosine-phosphorylated protein involved in cell spreading. Mol Biol Cell 2004; 15:787800.

25 Reutens AT, Begley CG. Endophilin-1: a multifunctional protein. Int J Biochem Cell Biol 2002; 34:1173-1177.

26 Jaworski J, Kapitein LC, Gouveia SM, et al. Dynamic microtubules regulate dendritic spine morphology and synaptic plasticity. Neuron 2009; 61:85-100.

27 Tomasoni R, Repetto D, Morini R, et al. SNAP-25 regulates spine formation through postsynaptic binding to p140Cap. Nat Commun 2013; 4:2136.

28 Gallop JL, Jao CC, Kent HM, et al. Mechanism of endophilin N-BAR domain-mediated membrane curvature. EMBO J 2006; 25:2898-2910.

29 Hotulainen P, Hoogenraad CC. Actin in dendritic spines: connecting dynamics to function. J Cell Biol 2010; 189:619-629.

30 Spector I, Shochet NR, Blasberger D, Kashman Y. Latrunculins--novel marine macrolides that disrupt microfilament organization and affect cell growth: I. Comparison with cytochalasin D. Cell Motil Cytoskeleton 1989; 13:127-144.

31 Vasquez RJ, Howell B, Yvon AM, Wadsworth P, Cassimeris L. Nanomolar concentrations of nocodazole alter microtubule dynamic instability in vivo and in vitro. Mol Biol Cell 1997; 
8:973-985.

32 Wu H, Reynolds AB, Kanner SB, Vines RR, Parsons JT. Identification and characterization of a novel cytoskeleton-associated pp60src substrate. Mol Cell Biol 1991; 11:5113-5124.

33 Uruno T, Liu J, Zhang P, et al. Activation of Arp2/3 complex-mediated actin polymerization by cortactin. Nat Cell Biol 2001; 3:259-266.

34 Hering H, Sheng M. Activity-dependent redistribution and essential role of cortactin in dendritic spine morphogenesis. $J$ Neurosci 2003; 23:11759-11769.

35 Iki J, Inoue A, Bito H, Okabe S. Bi-directional regulation of postsynaptic cortactin distribution by BDNF and NMDA receptor activity. Eur J Neurosci 2005; 22:2985-2994.

36 Di Stefano P, Damiano L, Cabodi S, et al. p140Cap protein suppresses tumour cell properties, regulating Csk and Src kinase activity. EMBO J 2007; 26:2843-2855.

37 Repetto D, Camera P, Melani R, et al. p140Cap regulates memory and synaptic plasticity through Src-mediated and citron-N-mediated actin reorganization. J Neurosci 2014; 34:1542-1553.

38 Hayashi K, Suzuki A, Hirai S, Kurihara Y, Hoogenraad CC, Ohno S. Maintenance of dendritic spine morphology by partitioning-defective $1 \mathrm{~b}$ through regulation of microtubule growth. J Neurosci 2011; 31:12094-12103.

39 Geraldo S, Khanzada UK, Parsons M, Chilton JK, Gordon-Weeks PR. Targeting of the F-actin-binding protein drebrin by the microtubule plus-tip protein EB3 is required for neuritogenesis. Nat Cell Biol 2008; 10:1181-1189.

40 Merriam EB, Millette M, Lumbard DC, et al. Synaptic regulation of microtubule dynamics in dendritic spines by calcium, F-actin, and drebrin. J Neurosci 2013; 33:16471-16482.
41 Barkho BZ, Song H, Aimone JB, et al. Identification of astrocyte-expressed factors that modulate neural stem/progenitor cell differentiation. Stem Cells Dev 2006; 15:407-421.

42 Li X, Barkho BZ, Luo Y, et al. Epigenetic regulation of the stem cell mitogen Fgf-2 by Mbd1 in adult neural stem/progenitor cells. J Biol Chem 2008; 283:27644-27652.

43 Lehtonen S, Zhao F, Lehtonen E. CD2-associated protein directly interacts with the actin cytoskeleton. Am J Physiol Renal Physiol 2002; 283:F734-743.

44 Banker G, Goslin K. Developments in neuronal cell culture. Nature 1988; 336:185-186.

45 Niu Y, Zhang C, Sun Z, et al. PtdIns(4)P regulates retromermotor interaction to facilitate dynein-cargo dissociation at the trans-Golgi network. Nat Cell Biol 2013; 15:417-429.

46 Sala C, Piech V, Wilson NR, Passafaro M, Liu G, Sheng M. Regulation of dendritic spine morphology and synaptic function by Shank and Homer. Neuron 2001; 31:115-130.

47 Zhang H, Macara IG. The polarity protein PAR-3 and TIAM1 cooperate in dendritic spine morphogenesis. Nat Cell Biol 2006; 8:227-237.

48 Carlin RK, Grab DJ, Cohen RS, Siekevitz P. Isolation and characterization of postsynaptic densities from various brain regions: enrichment of different types of postsynaptic densities. J Cell Biol 1980; 86:831-845.

49 Cho KO, Hunt CA, Kennedy MB. The rat brain postsynaptic density fraction contains a homolog of the Drosophila discslarge tumor suppressor protein. Neuron 1992; 9:929-942.

50 Bao L, Wang HF, Cai HJ, et al. Peripheral axotomy induces only very limited sprouting of coarse myelinated afferents into inner lamina II of rat spinal cord. Eur J Neurosci 2002; 16:175-185.

(Supplementary information is linked to the online version of the paper on the Cell Research website.) 